\title{
On a dynamical system with multiple chaotic attractors
}

\author{
XIAODONG LUO*, MARIUS-F. DANCA ${ }^{\dagger}$, MICHAEL SMALL* and GUANRONG CHEN ${ }^{\ddagger}$ \\ *Department of Electronic and Information Engineering, \\ Hong Kong Polytechnic University, Hong Kong, P.R. China \\ ${ }^{\dagger}$ Department of Mathematics, \\ Tehnofrig Technical College, 3400 Cluj-Napoca, Romania \\ ${ }^{\ddagger}$ Department of Electronic Engineering, \\ City University of Hong Kong, Hong Kong, P.R. China
}

March 18, 2013

\begin{abstract}
The chaotic behaviour of the Rabinovich-Fabrikant system, a model with multiple topologically different chaotic attractors, is analysed. Because of the complexity of this system, analytical and numerical studies of the system are very difficult tasks. Following the investigation of this system carried out in [Danca and Chen, 2004], this paper verifies the presence of multiple chaotic attractors in the system. Moreover, the Monte Carlo hypothesis test (or, equivalently, surrogate data test) is applied to the system for the detection of chaos.

Keywords: Rabinovich-Fabrikant system, multiple attractors, LIL algorithm, chaos detection, Monte Carlo hypothesis test, surrogate data test.
\end{abstract}

\section{Introduction}

Chaotic systems with multiple attractors have received increasing attention in recent years because of their great impacts on both theoretical analysis and engineering applications. Studies revealed that multi-attractors could be considered a source of unpredictability [Dutta et al., 1999], and they have some potential applications in, for instance, communications [Carroll and Pecora, 1998], mechanical dynamics [Lowenberg, 1998, Zhou et al., 2001], and ecology [Anand and Desrochers, 2004].

There are some, though not too many, lower-dimensional dynamical systems with multiple chaotic attractors. For example, a three-dimensional autonomous quadratic chaotic system, which has five equilibria and could produce two two-wing chaotic attractors, is designed and studied in [Lü et al., 2004]. As another example, recently reported in [Qi et al., 2005], a four-dimensional autonomous cubic system, generated through the so-called "proto-Lorenz systems", could yield four-wing chaotic attractors. More examples and studies can be found in [Chua et al., 1986, Han et al., 2005, Liu and Chen, 2004, Lü et al., 2003, Suykens and Vanderwalle, 1993, Yalcin et al., 2001]. 
A yet more particular example with multiple attractors is the one that will be further studied in this paper - the Rabinovich-Fabrikant system (called the "RF system" hereafter). This RF system arises from the practical demand of modelling the stochasticity due to the modulation instability in a nonequilibrium dissipative medium [Rabinovich and Fabrikant, 1979]. As indicated in Fig. 1, a pronounced chaotic behaviour can be observed from the autocorrelation function of the RF system versus the white noise with normal distribution. Compared to the ordinary multi-attractor systems, the RF system has a few interesting features. For instance, by examining the geometric shapes of the chaotic attractors in the phase space or the next relative maximum amplitudes, one may observe that some chaotic attractors exhibit quite different topological structures from the others ${ }^{1}$. Moreover, the system has proved a great challenge to many classical numerical methods (e.g., the Runge-Kutta method), since most of them may fail to integrate the model successfully for certain parameter values. For this reason, a special multi-step algorithm, the Local Iterative Linearization (LIL) method [Danca, 2005], is adopted in this paper. However, we will not perform a comparison of the performances between the LIL algorithm and the other standard numerical methods, since this is outside the scope of the present investigation.

More precisely, the focus of the present paper is to re-examine the chaotic behaviour of the RF system from a special point of view, with emphasis on the features of the multiple attractors and the topological differences between them.

In the endeavor of identifying chaotic behaviours, there are two kinds of strategies available in the literature. One strategy is to directly adopt some characteristic descriptions, for example the maximum Lyapunov exponent, the bifurcation diagrams, etc., in the belief that these reveal the essential features of chaotic behaviours. The other strategy is to conduct an investigation through the Monte Carlo null hypothesis test, or equivalently the surrogate data method [Theiler et al., 1992, Theiler, 1996]. In the latter approach, one actually tests against non-chaotic cases, which might be much simpler than a direct detection of the chaotic cases. In addition, with a proper (nonparametric) algorithm, one could generate a number of different realizations from the original data in test, which share the desired properties of the original data if the null hypothesis is true, but otherwise exhibit difference(s) in certain aspect(s) from the original data. With these data, even if one does not know the underlying process of the original test data a priori, and there is only a very limited amount of data, one could estimate the empirical distribution of the test statistic and the associated confidence interval under the null hypothesis, as will be shown later in the paper.

With the above motivations, in this paper we apply the surrogate data method to detect the chaotic behaviours of the RF system, which provides a good complement to the existing investigations using the conventional concepts like the Lyapunov exponent spectrum, histograms, Poincaré sections, first return map of the relative maximum, bifurcation diagrams, etc. [Danca and Chen, 2004].

The rest of the paper is organised as follows: In Section 2, a brief introduction is presented to describe the mathematical model of the RF system. A further investigation of the chaotic behaviours of the RF system is carried out in Section 3, based on the surrogate data method, where the multi-attractor feature

\footnotetext{
${ }^{1}$ An analytical proof remains to be a challenge for future work.
} 
of the RF system will also be discussed. Section 4 briefly concludes the investigation of the paper.

\section{The Rabinovich-Fabrikant system}

The Rabinovich-Fabrikant (RF) system is described by the following equations:

$$
\left\{\begin{array}{l}
\dot{x}_{1}=x_{2}\left(x_{3}-1+x_{1}^{2}\right)+a x_{1}, \\
\dot{x}_{2}=x_{1}\left(3 x_{3}+1-x_{1}^{2}\right)+a x_{2}, \\
\dot{x}_{3}=-2 x_{3}\left(b+x_{1} x_{2}\right),
\end{array}\right.
$$

with parameters $a, b>0$. This system models the dynamical behaviour arising from the modulation instability in a nonequilibrium dissipative medium [Rabinovich and Fabrikant, 1979].

The RF model (1) has the following five hyperbolic equilibria points:

$$
\begin{gathered}
X^{*}(0,0,0), \\
X_{1,2}^{*}\left( \pm x_{-},-\frac{b}{x_{-}}, 1-\left(1-\frac{a}{b}\right) x_{-}^{2}\right), \\
X_{3,4}^{*}\left( \pm x_{+},-\frac{b}{x_{+}}, 1-\left(1-\frac{a}{b}\right) x_{+}^{2}\right),
\end{gathered}
$$

with

$$
x_{ \pm}=\sqrt{1 \pm \sqrt{1-a b\left(1-\frac{3 a}{4 b}\right)} / 2\left(1-\frac{3 a}{4 b}\right)} .
$$

Denote the smooth real-valued function on the right-hand side of model (1) in the form of $f=$ $\left(f_{1}\left(x_{1}, x_{2}, x_{3}\right), f_{2}\left(x_{1}, x_{2}, x_{3}\right), f_{3}\left(x_{1}, x_{2}, x_{3}\right)\right)^{T}$. Then, it is easy to see that the system has the following symmetry:

$$
\begin{aligned}
& f_{1}\left(-x_{1},-x_{2}, x_{3}\right)=-f_{1}\left(x_{1}, x_{2}, x_{3}\right), \\
& f_{2}\left(-x_{1},-x_{2}, x_{3}\right)=-f_{2}\left(x_{1}, x_{2}, x_{3}\right), \\
& f_{3}\left(-x_{1},-x_{2},-x_{3}\right)=-f_{3}\left(x_{1}, x_{2}, x_{3}\right),
\end{aligned}
$$

which influences the geometry of the attractors. In addition, the divergence of the system is

$$
\nabla \cdot f\left(x_{1}, x_{2}, x_{3}\right)=\sum_{i=1}^{3} \frac{\partial}{\partial x_{i}} f_{i}\left(x_{1}, x_{2}, x_{3}\right)=2(a-b),
$$

which implies that the system is dissipative for $a<b$, since the volume of the system contracts according to the Liouville formula.

Notice that the system behaviour is very sensitive to the parameter $b$. Therefore, for most cases discussed in [Danca and Chen, 2004] the parameter $a$ is fixed 0.1 , with the parameter $b$ being in the interval $(0.13,1.3)$. As supplementary examples, we do consider a few cases with $a \neq 0.1$ 
and $b \notin(0.13,1.3)$. Specifically, the parameters values $(a, b)$ to be re-examined in this work include: $\left\{(0.1,0.2715),(0.1,0.2876),(0.1,0.98),(0.1,1.215),(0.05,0.06),(-1,-0.2)^{2}\right\}$. For more details, see [Danca and Chen, 2004].

\section{Chaotic behaviours of the RF system}

As mentioned before, the presence of multiple equilibrium points and cubic polynomial terms in the mathematical model of the RF system yields very complicated and spectacular dynamical phenomena. In addition, the inherent difficulty in numerical integration obscures the accuracy of the analytical studies. Therefore, it is almost impossible to obtain a stable trajectory due to numerical errors, while the RF system behaves chaotically (see Fig. 2, where two stable periodic motions are presented). The situation changes in the cases of some chaotic attractors of the RF model, where "false chaos" could be generated via numerical errors.

Consequently, a question naturally arises: are the numerically obtained attractors really chaotic as reported in the literature, or are they simply some false replicas?

To answer this question, the surrogate data method [Theiler et al., 1992, Theiler, 1996, Small, 2005] is employed. As discussed in the literature, the elements that form the framework of a surrogate test are null hypothesis, surrogate generation algorithm, discriminating statistic, and discriminating criterion. Since, in the present case, the pseudo-periodic time series is either chaotic or periodic but contaminated with dynamical noise (round errors, for example), the following null hypothesis is formulated: The pseudo-periodic time series was generated from a periodic orbit perturbed by uncorrelated dynamical noise, while the alternative hypothesis is that the time series is chaotic, and the potential risk is considered as the false rejection rate, which will be analysed below.

The method for surrogate generation follows the pseudo-periodic surrogate (PPS) algorithm [Small et al., 2001, Small, 2005]. As a popular choice, the correlation dimension [Grassberger and Procaccia, 1983] is chosen as the discriminating statistic here, while the adopted discriminating criterion is extended from the nonparametric method proposed in [Theiler and Prichard, 1997]. Specifically, the discriminating criterion examines the ranks of the statistic values of the original time series and its surrogates (for this reason, we call it ranking criterion hereafter). Suppose that the discriminating statistic of the original data is $T_{0}$ and the surrogate values are $\left\{T_{i}\right\}_{i=1}^{N}$, given $N$ surrogate realizations. Then, one sorts the set $\left\{T_{0}, T_{1}, \ldots, T_{N}\right\}$ in ascending order. Let the rank of $T_{0}$ be denoted by $R_{0}$, and the median of the set $\{1,2, \ldots, N+1\}$ be denoted by $M$. For one-sided tests, if $R_{0}<M$, the false rejection rate is $R_{0} /(N+1)$ to reject the null hypothesis; otherwise, it is $\left(N+2-R_{0}\right) /(N+1)$. For two-sided tests, however, if $R_{0}<M$ then the rate is thought to be $2 R_{0} /(N+1)$ to reject the null hypothesis; otherwise, it is estimated to be $2\left(N+2-R_{0}\right) /(N+1)$.

In the following, we apply the above framework to re-examine several chaotic cases reported in [Danca and Chen, 2004]. In all the tests, the time series measured from the first coordinate $x_{1}$ will be

\footnotetext{
${ }^{2}$ The last case is interesting only from a mathematical point of view, since physically $a$ and $b$ must be positive.
} 
considered, and the PPS algorithm will be applied to produce 100 surrogates. For numerical computation of the correlation dimensions, it is recommended to adopt the Gaussian kernel algorithm (GKA) [Diks, 1996, Yu, 2000] because of its efficiency and robustness against noise. But, to speed up the calculation, only 2000 data points are used as the reference points in computation. Therefore, there are some statistical fluctuations even for the same data set in calculating its correlation dimension. For this reason, the correlation dimension of the original time series will be calculated 100 times to estimate the mean value and the standard deviation. For the 100 surrogates, however, they will only be calculated once for simplicity. These test results will be plotted together into the related plots (see, for example, Fig. 4, Fig. 7, Fig. 10, and so on), where the middle lines denote the mean correlation dimensions for 100 times calculations of the original time series, and the upper and lower lines represent the values that are three times the standard deviation away from the mean correlation value. Finally, the correlation dimensions of the 100 surrogates are marked by the asterisks.

Case 1: $a=0.1$ and $b=0.2715$

The LIL algorithm is adopted to integrate the RF model with step size $h=0.005$. After discarding the transients, the attractor $A_{1}$ in the three-dimensional phase space and the corresponding plane projections are plotted in Fig. 3.

Based on the computation results illustrated in Fig. 4, it is found that the rank of the mean correlation dimension of the original data is 94 among all of the 100 values. Therefore, according to the ranking criterion introduced previously, the possible false rate to reject the null hypothesis is considered roughly $8 \%$ for one-sided test, or $16 \%$ for two-sided test ${ }^{3}$. This implies that the data in test is more likely to be chaotic compared with the possibility of the data in test being periodic with dynamical noise.

Remark: The above procedure will be applied in the same way to interpret the test results for all the other cases below, therefore it will not be described explicitly therein.

Case 2: $a=0.1$ and $b=0.2876$

In this case, the RF system is integrated with step size $h=0.005$. The three-dimensional attractor $A_{2}$ and its projections are depicted in Fig. 6.

The procedure of null hypothesis test is the same as above. After generating 100 surrogates, their correlation dimensions, together with that of the original data, are calculated and plotted in Fig. 7. Among these values, the mean correlation dimension of the original data is ranked 89. Thus, the estimated rate of a possible false rejection is roughly $13 \%$ for one-sided test (or $26 \%$ for two-sided test) according to the ranking criterion. This value, compared to the above result, indicates that the discrimination power of the correlation dimension is significantly reduced in this case.

\footnotetext{
${ }^{3}$ In other words, the probability that we are correct to reject the null hypothesis based on the ranking criterion is $92 \%$ for one-sided test, or $84 \%$ for two sided test.
} 
Case 3: $a=0.1$ and $b=0.98$

Here, the sampling step is set to $h=0.03$. The obtained results for the corresponding attractor $A_{3}$ are illustrated in Fig. 9.

With respect to the surrogate data test, numerical computations (see Fig. 10) indicate that in this case the discrimination power of the correlation dimension increases: The mean correlation dimension of the original data ranks 85 together with the other 100 values of the surrogates. Therefore, the possible false rejection rate is $17 \%$ for one-sided test (or $34 \%$ for two-sided test).

Case 4: $a=0.1$ and $b=1.215$

In this case, the system possess two different attractors with different initial conditions, which lie in two different attraction basins [Danca and Chen, 2004]. Thus, one attractor originates from the point $(-0.5,6,1.1)$, while the other is born out at $(0.5,-6,1.1)$. These two attractors, $A_{4}$ and their twodimensional projections obtained with $h=0.008$, are plotted in Fig. 12.

Surrogate tests on the time series of both attractors lead to the same result. Here, we only present the result with initial condition $(-0.5,6,1.1)$. As shown in Fig. 13, the mean correlation dimension ranks the highest among all values; therefore, the corresponding false rejection rates are considered as $1 \%$ for one-sided test (or $2 \%$ for two-sided test) according to the ranking criterion.

Case 5: $a=0.05$ and $b=0.06$

In this case, the numerical integration is realised with $h=0.005$. The generated attractor $A_{5}$ and its projections are indicated in Fig. 15.

The PPS algorithm is applied again to produce 100 surrogates, while the GKA is used to calculate the correlation dimensions of the original data and the surrogates. Numerical evaluation reveals that the correlation dimension has the same discrimination power as the above case, i.e., the mean correlation dimension of the original data ranks the highest again among the ensemble of correlation dimensions (see Fig. 16). Therefore, the test will bear the same possible false rejection rate(s) as the previous case.

Case 6: $a=-1$ and $b=-0.1$

The last case to be examined is sampled at the rate of $h=0.005$. The corresponding attractor $A_{6}$ is shown in Fig. 18.

In this case, the correlation dimension also suffers from the problem of fluctuation of the discrimination power. In fact, as can be seen from Fig. 19, the mean correlation dimension of the original data ranks 93 among the ensemble values. Hence, the highest false rejection rate is around $9 \%$ for one-sided test, or $18 \%$ for two-sided test.

As can be seen from the above figures, the system may exhibit the following dynamical behaviours: 
- A double-saddle or a single double-wing chaotic attractor (Fig. 6),

- Coexistence of chaotic attractors (Fig. 12),

- Saddle (Fig. 3 and Fig. 15),

- "Gramophon"-like chaotic attractors (Fig.18).

In Fig. 21, the two-dimensional collection of the considered multiple chaotic attractors is presented. With the exception of the attractors $A_{3}$ and $A_{4}$, the rest of the attractors belong to different regions of the phase space.

The topological difference between all these attractors can also be observed from the pronounced differences between their first return maps of the relative maximum amplitude (see Figs. 5, 8, 11, 14, 17, and 20). As is well known, these thin sheets are almost one-dimensional maps, from which chaos can be predicted.

It is interesting to note that usually the maps like those plotted in Figs. 11 and 14 may produce intermittency. Another intriguing aspect is the resemblance between these maps and those of some well-known attractors. For example, the first return map of the case of $a=0.1$ and $b=0.2715$ reminds the first return map of the Belusov-Zhabotinskii reaction [Roux et al., 1982] (the same for their corresponding chaotic attractors - $A_{4}$ for RF system), the map of the case of $a=0.1$ and $b=0.98$ is analogous to that of the Lorenz system, while the case of $a=0.1$ and $b=1.215$ is reminiscent of the Rössler system.

\section{Conclusions}

In this paper, we have given a brief review of the chaotic behaviour of the Rabinovich-Fabrikant system. As reported in the literature, this particular system has many interesting features. In this work, we have illustrated that the system may possess multiple topologically different chaotic attractors.

The focus of this paper is two-folded: First, it is to investigate the chaotic behaviour of the RF system through the method of Monte Carlo hypothesis test [Small et al., 2001, Small, 2005, Theiler et al., 1992, Theiler, 1996], which could be considered as an alternative test strategy to the conventional ones (see [Danca and Chen, 2004]). With this method, we have examined the hypothesis which assumes that the reported multiple attractors are not chaotic. Numerical calculations have indicated that, in all cases, this assumption is not favorable, which (indirectly) provides supportive evidence of their chaotic behaviour reported in [Danca and Chen, 2004]. Second, it is to indicate that some chaotic attractors may appear quite differently from the others, from a topological point of view. This phenomenon has been illustrated through the phase-space plots and the first return maps of the relative maximum amplitudes. In summary, we believe that the RF system does have multiple chaotic attractors. 


\section{Acknowledgement}

$\mathrm{XL}$ is supported by a direct allocation grant from the Hong Kong Polytechnic University; GRC is supported by the Hong Kong Research Grants Council under the CERG Grant CityU 1114/05E.

\section{References}

[Anand and Desrochers, 2004]

[Carroll and Pecora, 1998]

[Chua et al., 1986]

[Chen and Ueta, 1999]

[Danca and Chen, 2004]

[Danca, 2005]

[Diks, 1996]

[Dutta et al., 1999]

[Grassberger and Procaccia, 1983]

[Han et al., 2005]

[Henson et al., 2002]
Anand, M. \& Desrochers, R. E. [2004] "Quantification of restoration success using complex systems concepts and models," Restoration Ecology, 12(1): 117-123.

Carroll, T. L. \& Pecora, L. M. [1998] "Using multiple attractor chaotic systems for communication," Proc. ICECS'1998.

Chua, L. O., Komuro, M. \& Matsumoto, T. [1986] " The double scroll family," IEEE Trans. Circuits Syst. -I 33, 1072-1118.

Chen, G. \& Ueta, D. [1999] "Yet another chaotic attractor," Int. J. Bifurcation and Chaos 9, 1465-1466.

Danca, M. -F. \& Chen, G. [2004] "Bifurcation and chaos in a complex model of dissipative medium," Int. J. Bifurcation and Chaos 14, 3409-3447.

Danca, M.-F. [2005] "A multistep algorithm for ODEs," Dynamics of Continuous, Discrete Impulsive and Systems Series B: Applications and Algorithms, accepted.

Diks, C. [1996] "Estimating invariants of noisy attractors," Phys. Rev. E 53(R), 4263-4266.

Dutta, M. Nusse, H. E., Ott, E. \& Yorke, J. A. [1999] "Multiple attractor bifurcations: a source of unpredictability in piecewise smooth systems," arXiv.org/chao-dyn/chao-dyn/9904017

Grassberger, P. \& Procaccia, I. [1983] "Characterization of strange attractors," Phys. Rev. Lett. 50, 346-349.

Han, F., Yu, X., Lü, J., Chen, G. \& Feng, Y. [2005] "Generating multi-scroll chaotic attractors via a linear second-order hysteresis System," Dynamics of Continuous, Discrete and Impulse Systems Series B: Applications and Algorithms 12, 95-110.

Henson, S. M., Costantino, R. F., Desharnais, R. A., Cushing, J. M. \& Dennis, B. [2002] "Basins of attraction: population dynamics with two stable 4-cycles," OIKOS 98: 17-24. 
[Liu and Chen, 2004]

[Lowenberg, 1998]

[Lü et al., 2003]

[Lü et al., 2004]

[Qi et al., 2005]

[Roux et al., 1982]

[Small et al., 2001]

[Small, 2005]

[Suykens and Vanderwalle, 1993]

[Theiler et al., 1992]

[Theiler, 1996]
Liu W. \& Chen, G. [2004] " Can a three-dimensional smooth autonomous quadratic chaotic system generate a single four-scroll attractor?" Int. J. Bifurcation and Chaos 14: 1395-1403.

Lowenberg, M. H. [1998] "Bifurcation analysis of multiple-attractor flight dynamics," Philosophical Transactions of the Royal Society A: Mathematical, Physical and Engineering Sciences, 356(1745): 22972319.

Lü, J., Yu, X. \& Chen, G. [2003] "Generating chaotic attractors with multiple merged basins of attraction: A switching piecewise-linear control approach," IEEE Trans. Circuits Syst. -I 50, 198-207.

Lü, J., Chen, G. \& Cheng, D. [2004] "A new chaotic system and beyond: The generalized Lorenz-like system," Int. J. Bifurcation and Chaos 14, 1507-1537.

Qi, G., Du, S., Chen, G., Chen, Z. \& Yuan, Z. [2005] "On a four-dimensional Chaotic System," Chaos, Solitons and Fractals 23, 1671-1682.

[Rabinovich and Fabrikant, 1979] Rabinovich, M. I. \& Fabrikant, A. L. [1979] "Stochastic selfmodulation of waves in nonequilibrium media," J. E. T. P. (Sov .) 77, 617-629.

Roux, J. C., Turner, J. S., McCormick, W. D. \& Swinney, H. L. [1982] "Experimental observations of complex dynamics in a chimical reaction," in Non-linear Problems: Present and Future. Ed. Bishop A. R., Campbell, D. K. and Nicolaenko B. (Amsterdam, NorthHolland).

Small, M., Yu, D. J. \& Harrison, R. G. [2001] "A surrogate test for pseudo-periodic time series data," Phys. Rev. Lett. 87: 188101.

Small, M. [2005] Applied Nonlinear Time Series Analysis: Applications in Physics, Physiology and Finance (World Scientific).

Suykens, J. A. K \& Vandewalle, J. [1993] "Generation of n-double Scrolls (n=1, 2, 3, 4,...)," IEEE Trans. Circuits Syst. -I 40, 861-867.

Theiler, J., Eubank, S., Longtin, A., Gaidrikian, B. \& Farmer, J. D. [1992] "Testing for nonlinearity in time series: the method of surrogate data," Physica D 58, 77-94.

Theiler, J. \& Prichard, D. [1996] "Constrained-realization MonteCarlo method for hypothesis testing," Physica D 94, 221-235. 
[Theiler and Prichard, 1997]

[Yalcin et al., 2001]

[Yu, 2000]

[Zhou et al., 2001]
Theiler, J. \& Prichard, D. [1997] "Using 'surrogate surrogate Data' to calibrate the actual rate of false positives in tests for nonlinearity in time series Fields," Inst. Commun. 11, 99-112.

Yalcin, M. E., Ozoguz, S., Suykens, J. A. K., \& Vandewalle, J. [2001] "n-scroll chaos generators: A simple circuit model," Electron. Lett. 37, 147-148.

Yu, D. J., Small, M., Harrison, R. G. and Diks, C. [2000] "Efficient implementation of the Gaussian kernel algorithm in estimating invariants and noise level from noisy time series data," Phys. Rev. E 61, 3750-3756.

Zhou, N.-F., Luo, J.-W. \& Cai, Y.-J. [2001] "Implementation and simulation of chaotic behavior of multi-attractor generated by a physical pendulum" (in Chinese), J. of Zhejiang University, 28(1): $42-45$. 


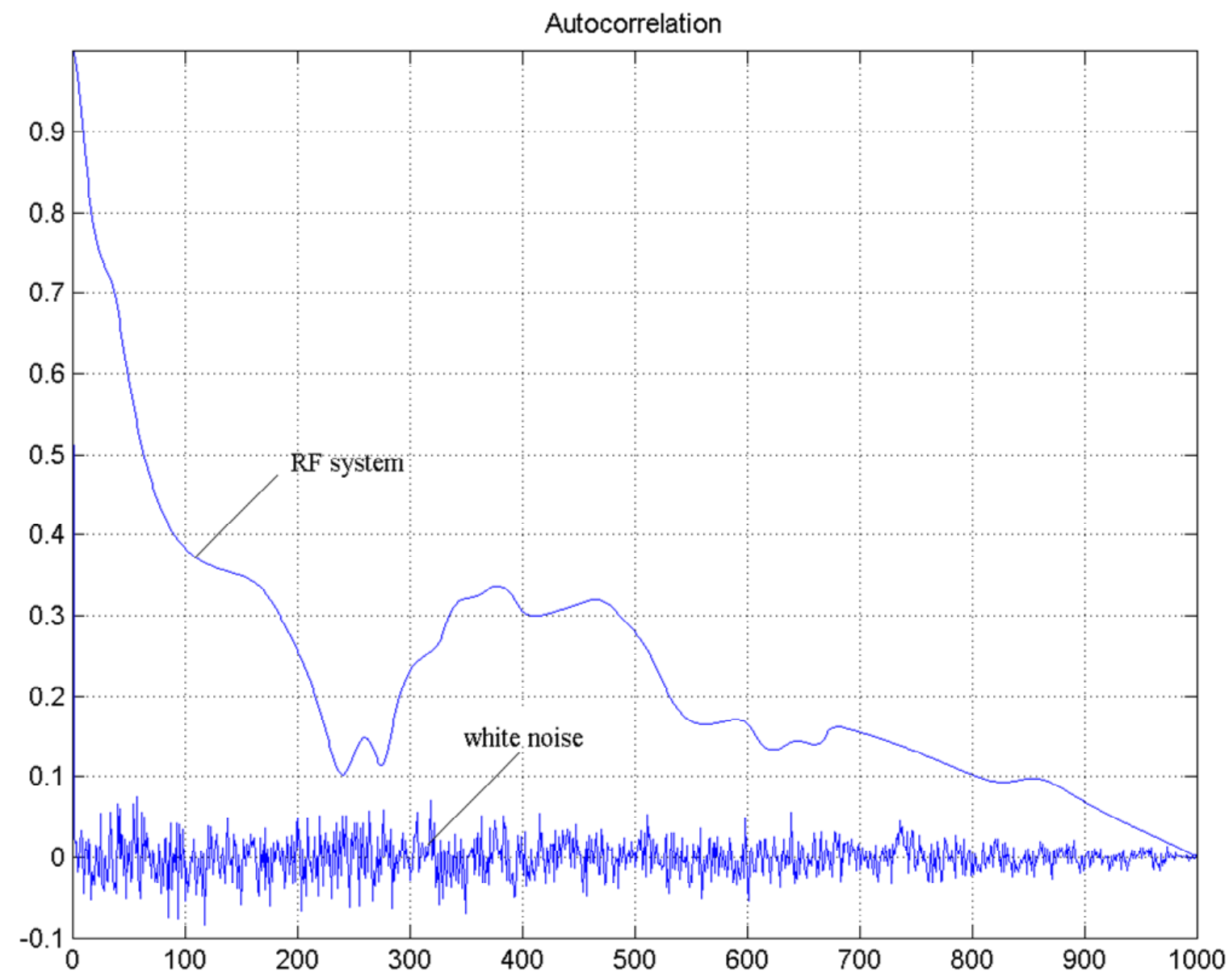

Figure 1: The autocorrelation functions of the RF system and that of the normal Gaussian white noise. 


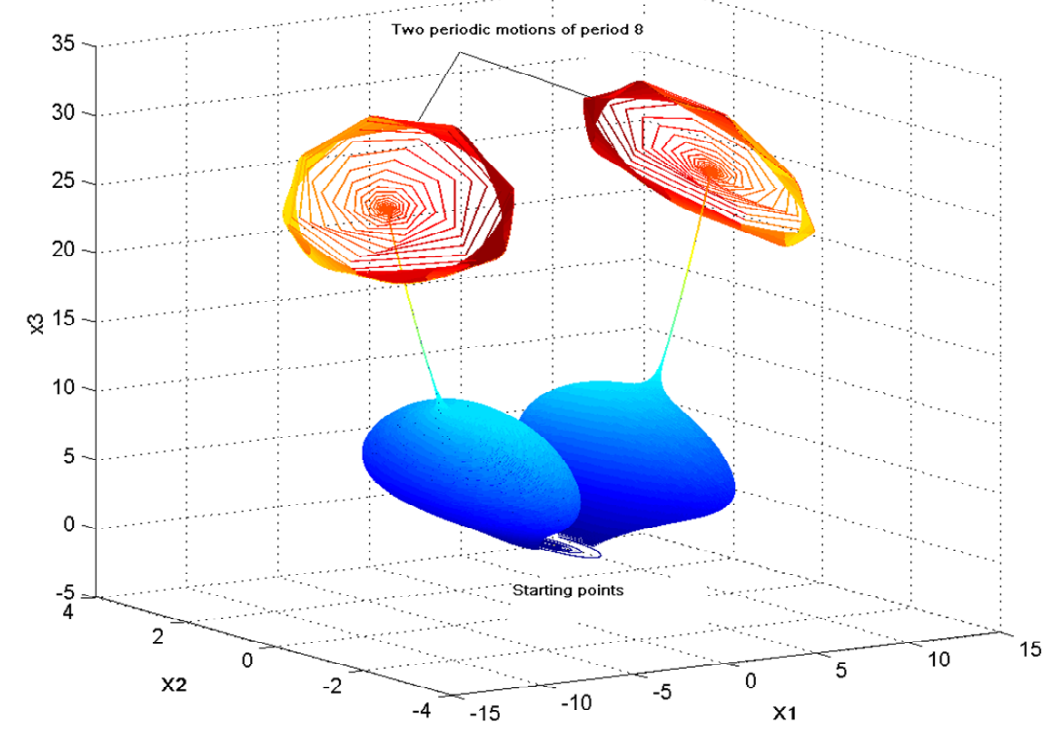

Figure 2: Two periodic motions of period 8 for the case of $a=0.1$ and $b=0.05$. 
(a)

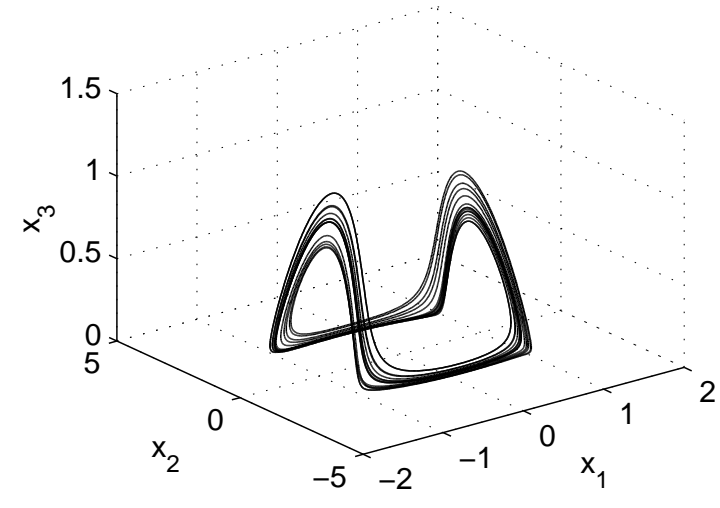

(c)

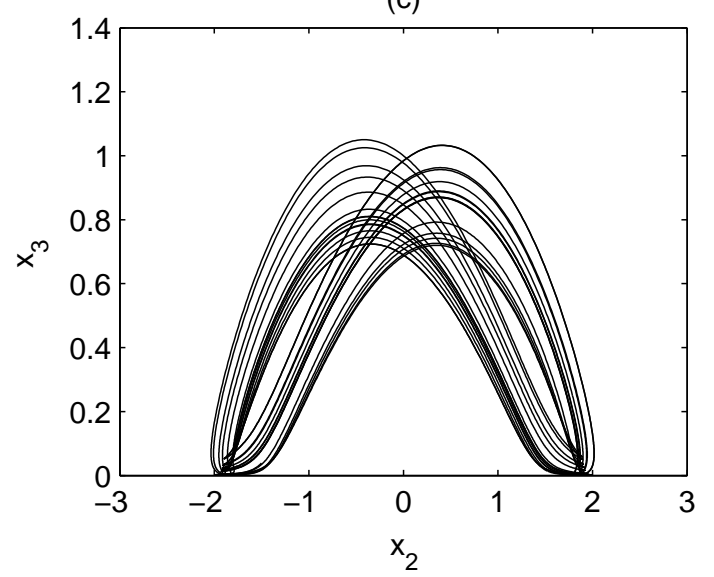

(b)

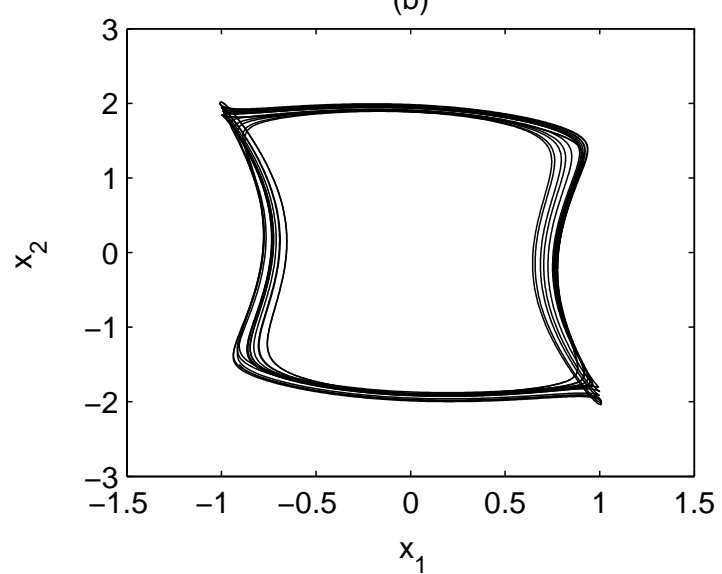

(d)

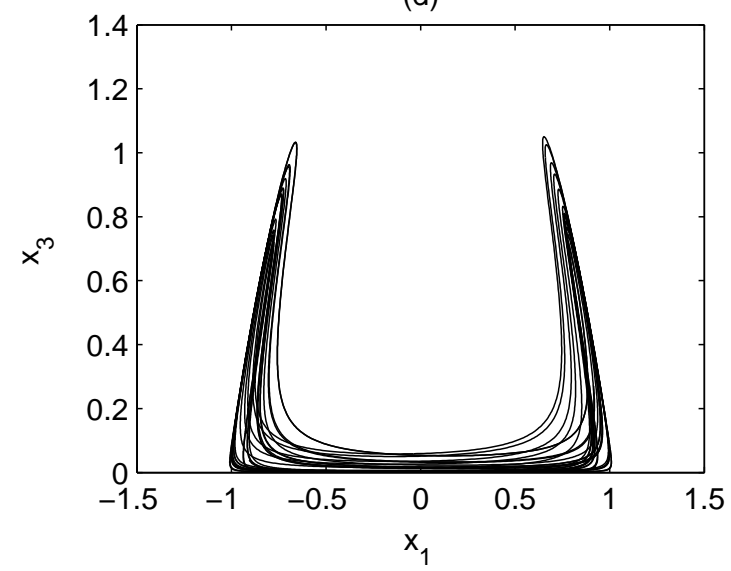

Figure 3: The chaotic attractor $A_{1}$ for the case of $a=0.1$ and $b=0.2715$. (a) Three-dimensional view; (b)-(d) Two-dimensional views. 


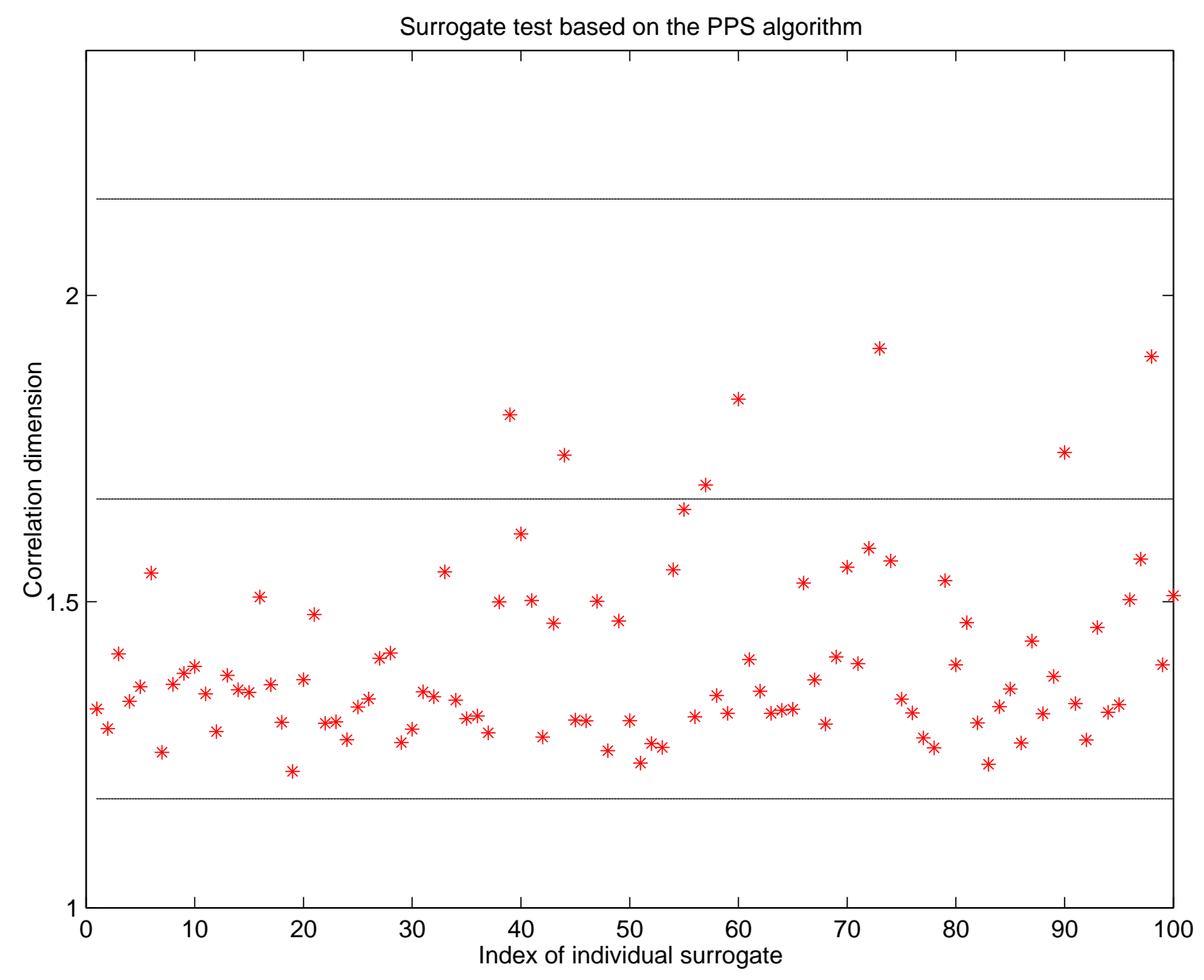

Figure 4: Surrogate test based on the PPS algorithm for the case of $a=0.1$ and $b=0.2715$. The middle line is the average correlation dimension over 100 calculations of the original time series, while the upper and lower lines denote the values that are thrice the standard deviation away from the average value. The correlation dimensions of the 100 surrogates are marked as asterisks. 


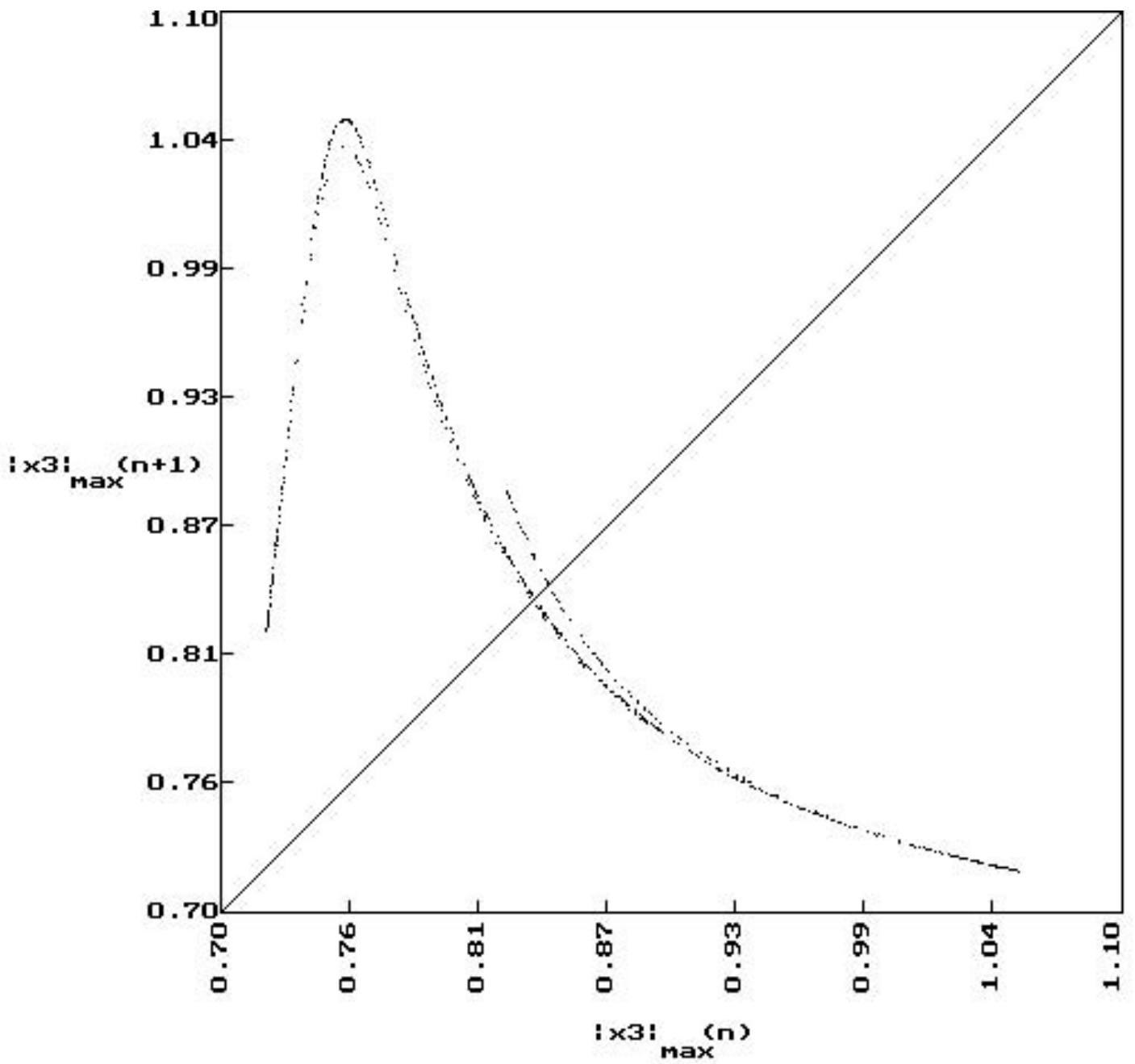

Figure 5: Next amplitude plot of the relative maximums for the case of $a=0.1$ and $b=0.2715$ (taken from [Danca \& Chen 2004). 
(a)

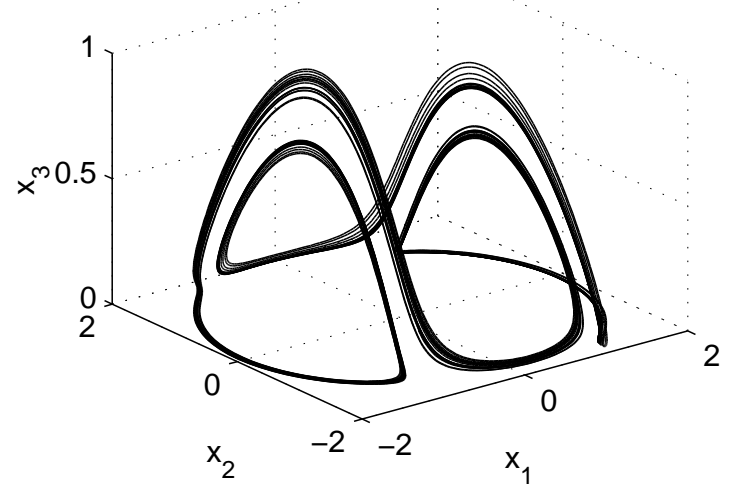

(c)

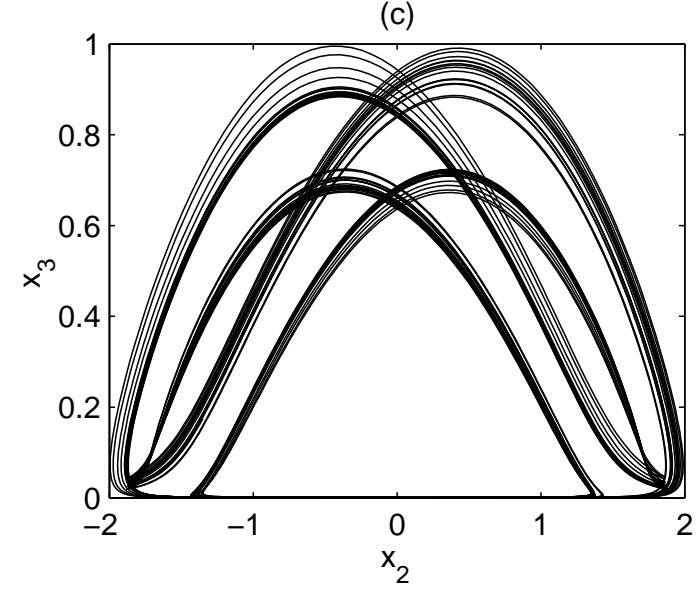

(b)

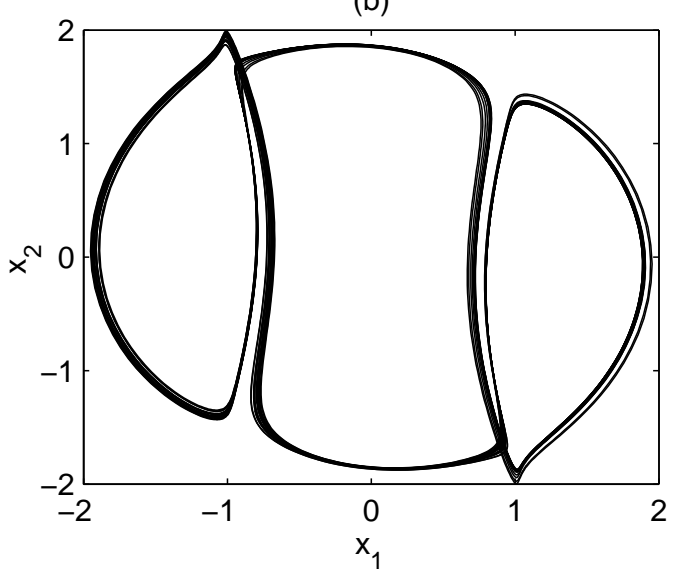

(d)

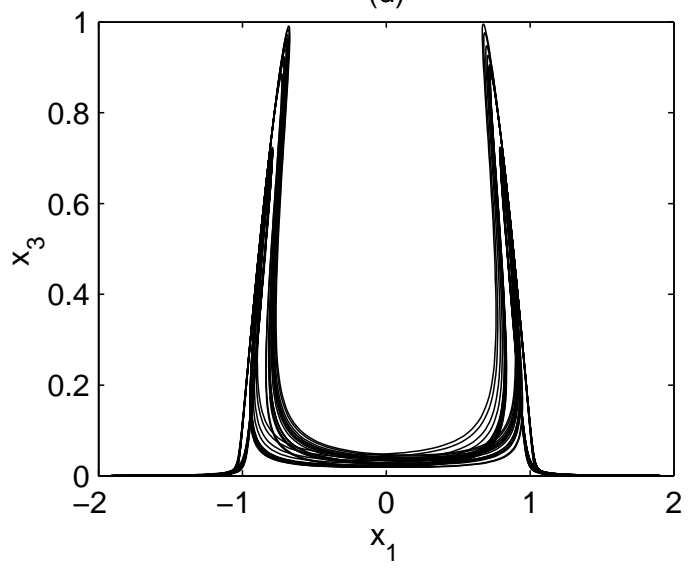

Figure 6: The chaotic attractor $A_{2}$ for the case of $a=0.1$ and $b=0.2876$. (a) Three-dimensional view; (b)-(d) Two-dimensional views. 


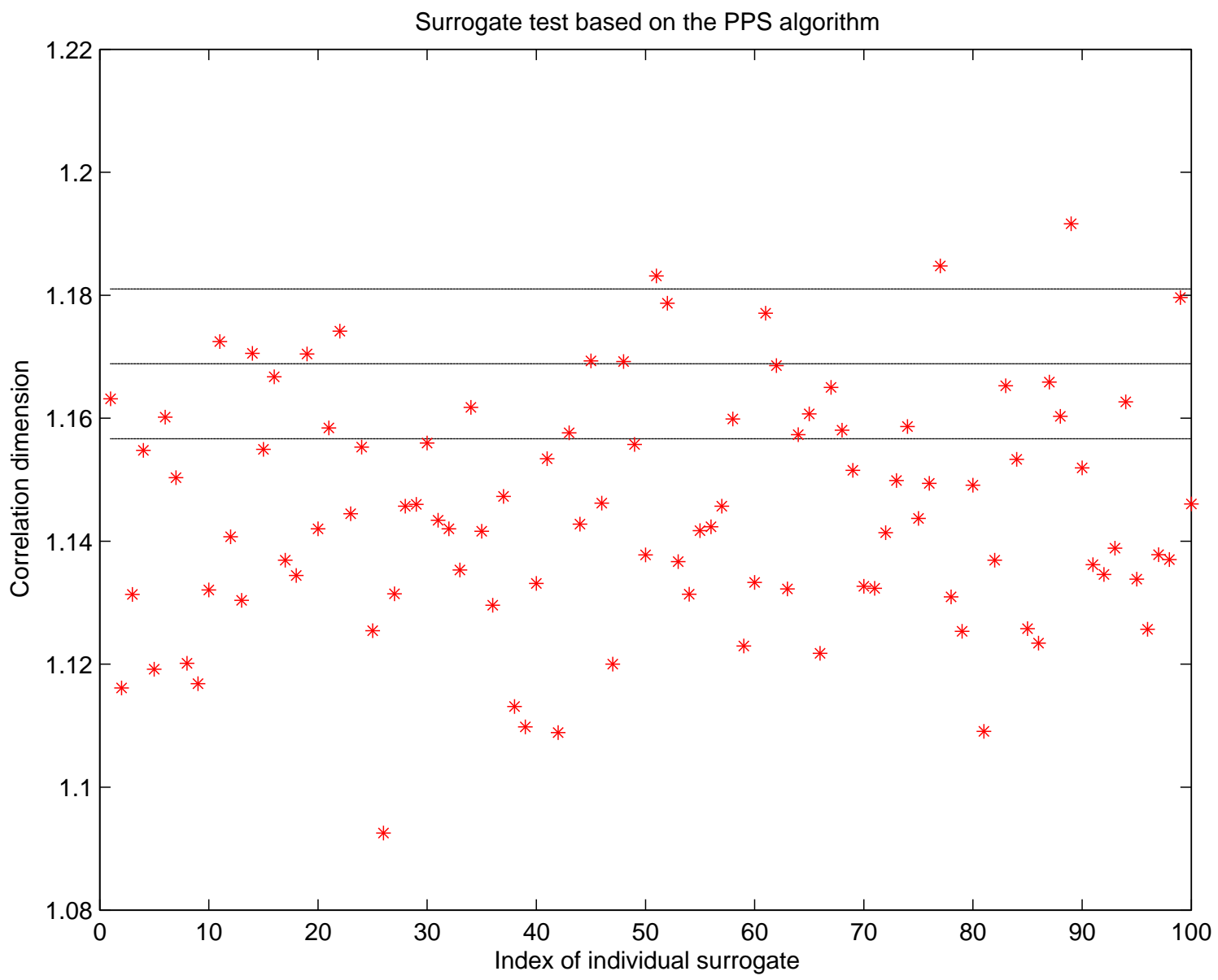

Figure 7: Surrogate test based on the PPS algorithm for the case of $a=0.1$ and $b=0.2876$. The meaning of the lines and asterisks are the same as that in Fig. 4. 


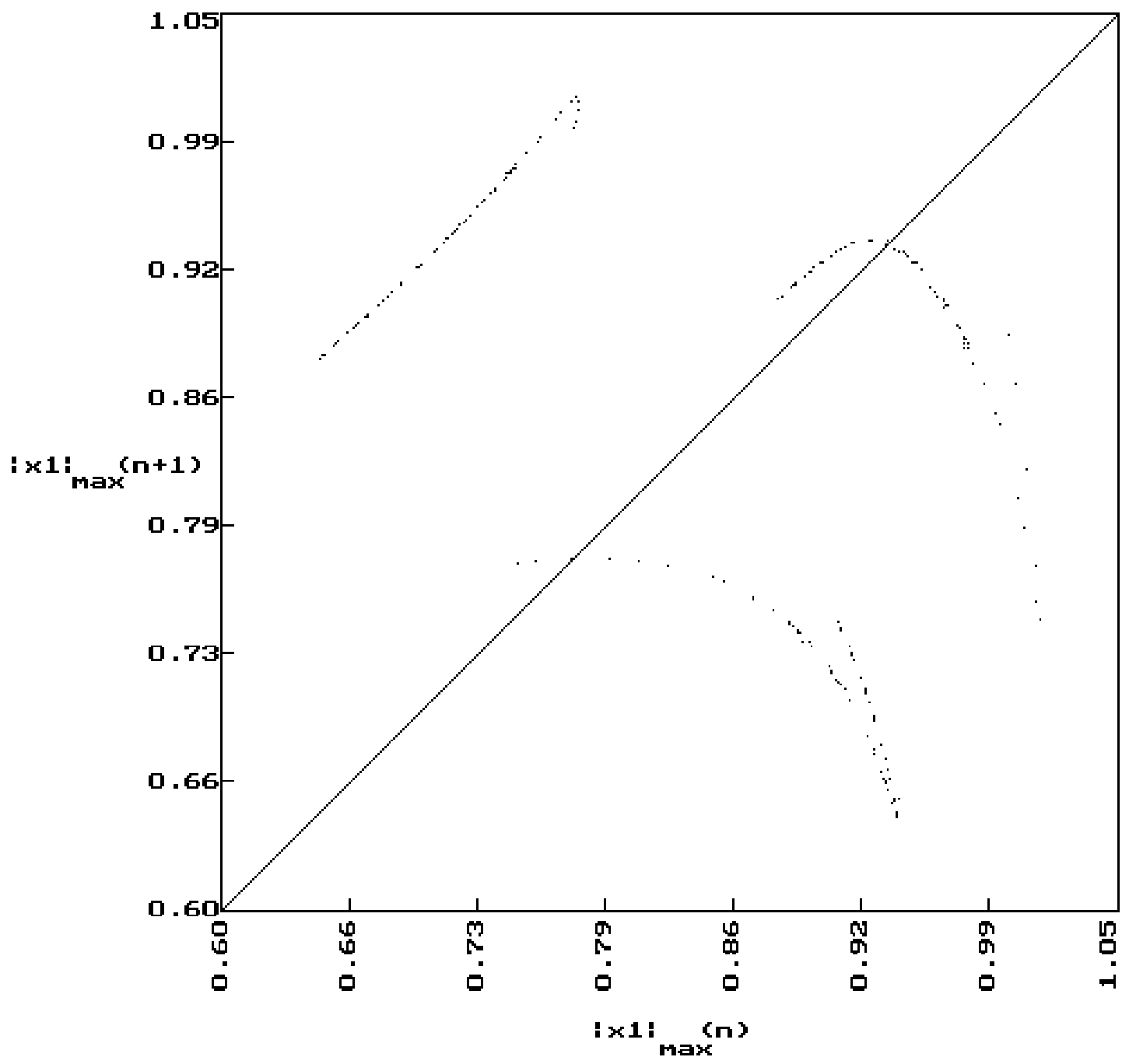

Figure 8: Next amplitude plot of the relative maximums for the case of $a=0.1$ and $b=0.2876$ (taken from [Danca \& Chen 2004]). 
(a)

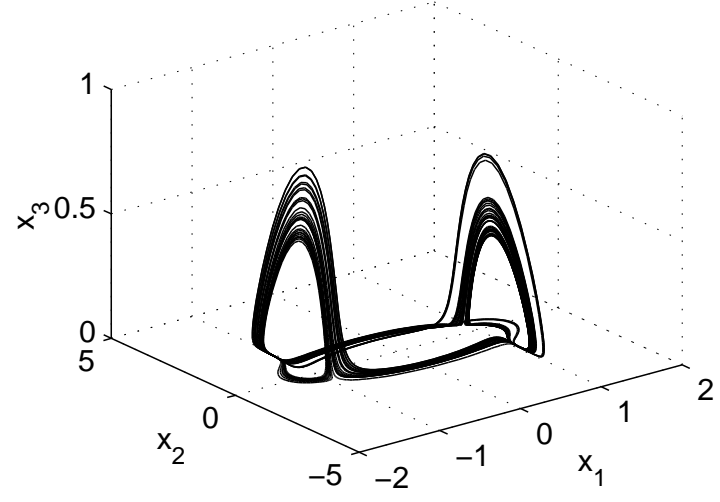

(c)

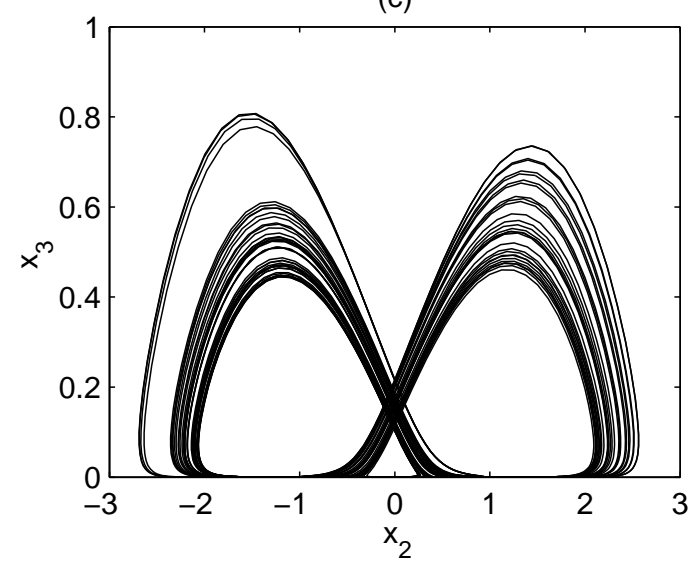

(b)

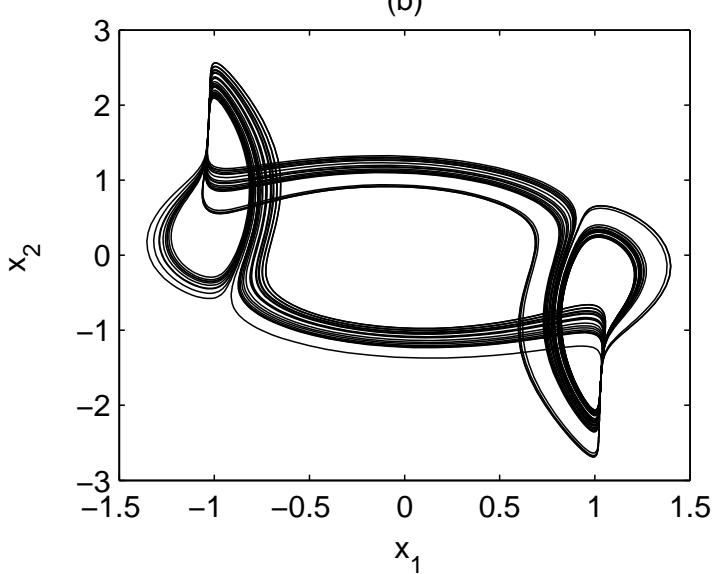

(d)

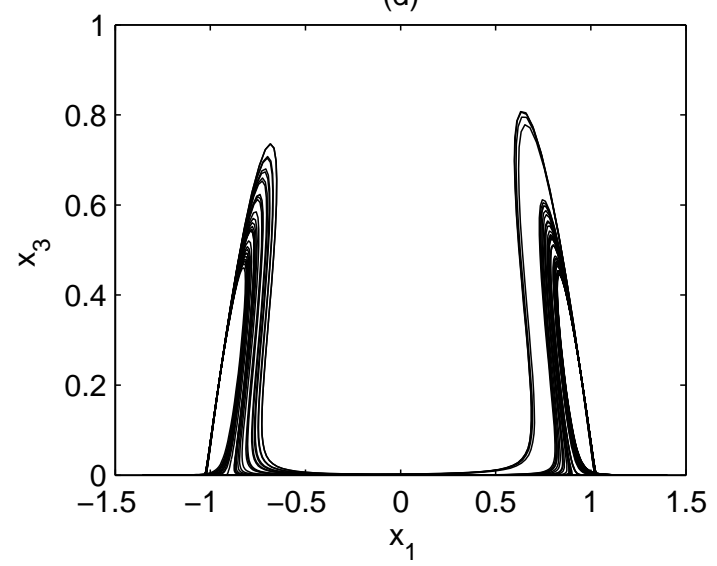

Figure 9: The chaotic attractor $A_{3}$ for the case of $a=0.1$ and $b=0.98$. (a) Three-dimensional view; (b)-(d) Two-dimensional views. 


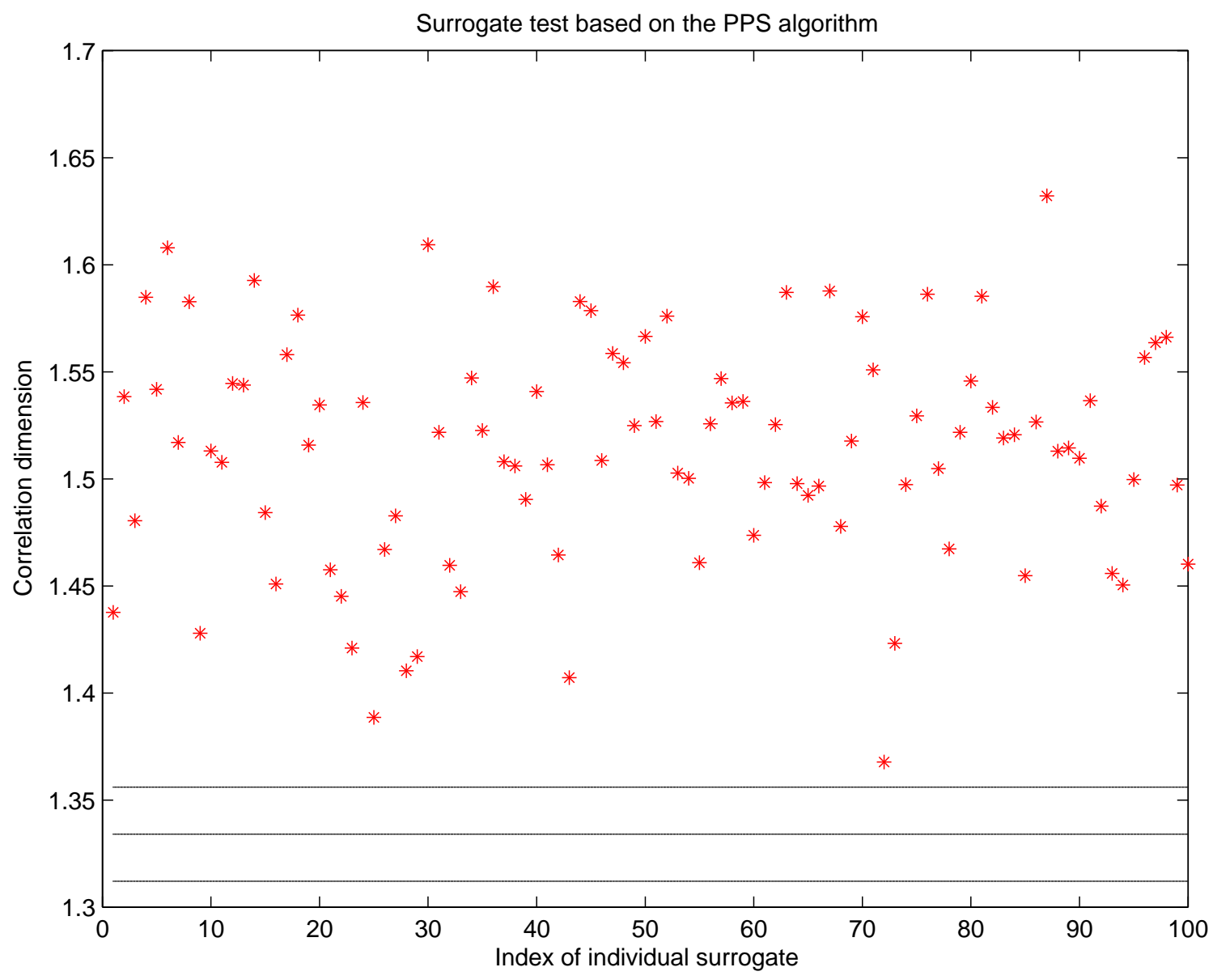

Figure 10: Surrogate test based on the PPS algorithm for the case of $a=0.1$ and $b=0.98$. The meaning of the lines and asterisks are the same as that in Fig. 4. 


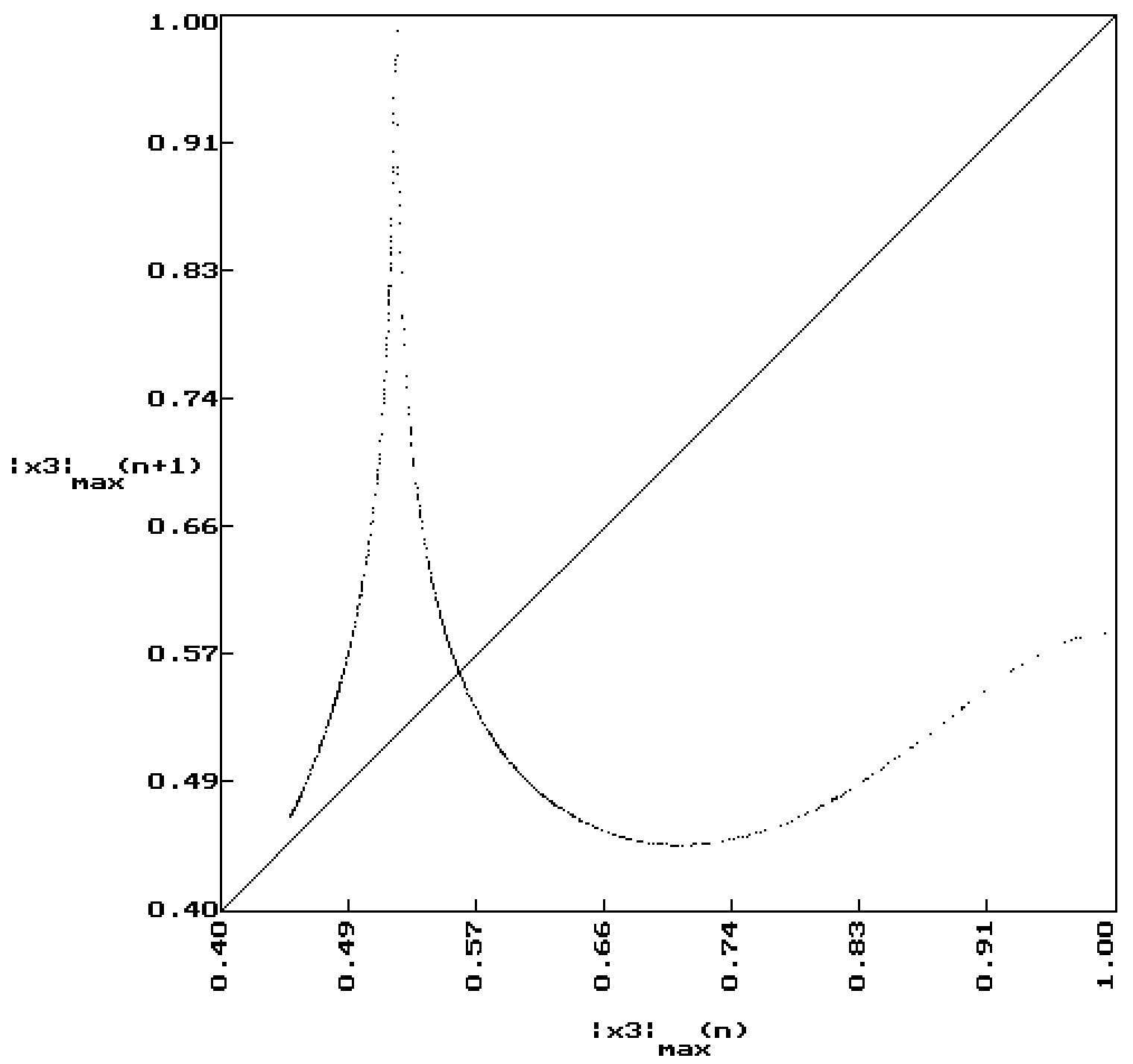

Figure 11: Next amplitude plot of the relative maximums for the case of $a=0.1$ and $b=0.98$ (taken from [Danca \& Chen 2004]). 
(a)

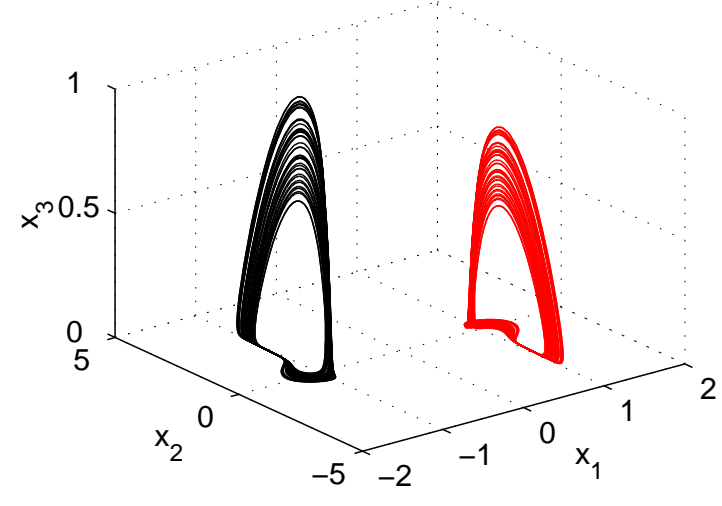

(c)

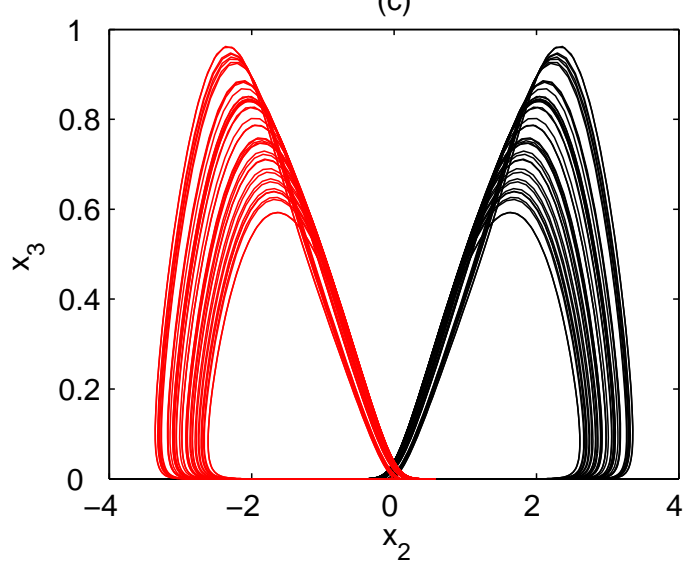

(b)

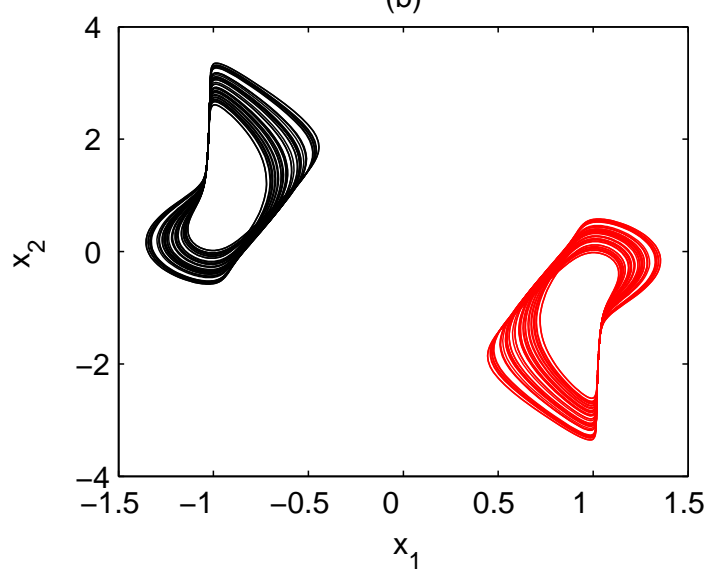

(d)

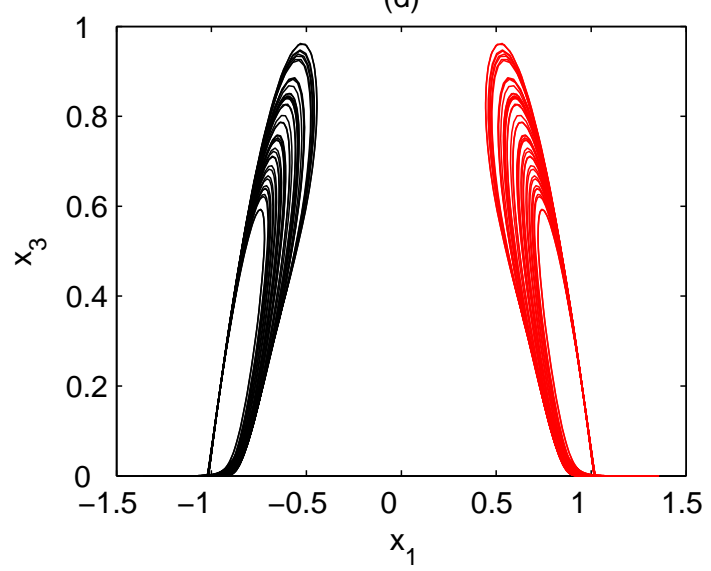

Figure 12: The chaotic attractors $A_{4}$ of the RF system for the case of $a=0.1$ and $b=1.215$ with initial conditions $(-0.5,6,1.1)$ (in black) and $(0.5,-6,1.1)$ (in red) (a) Three-dimensional view; (b)-(d) Two-dimensional views. 


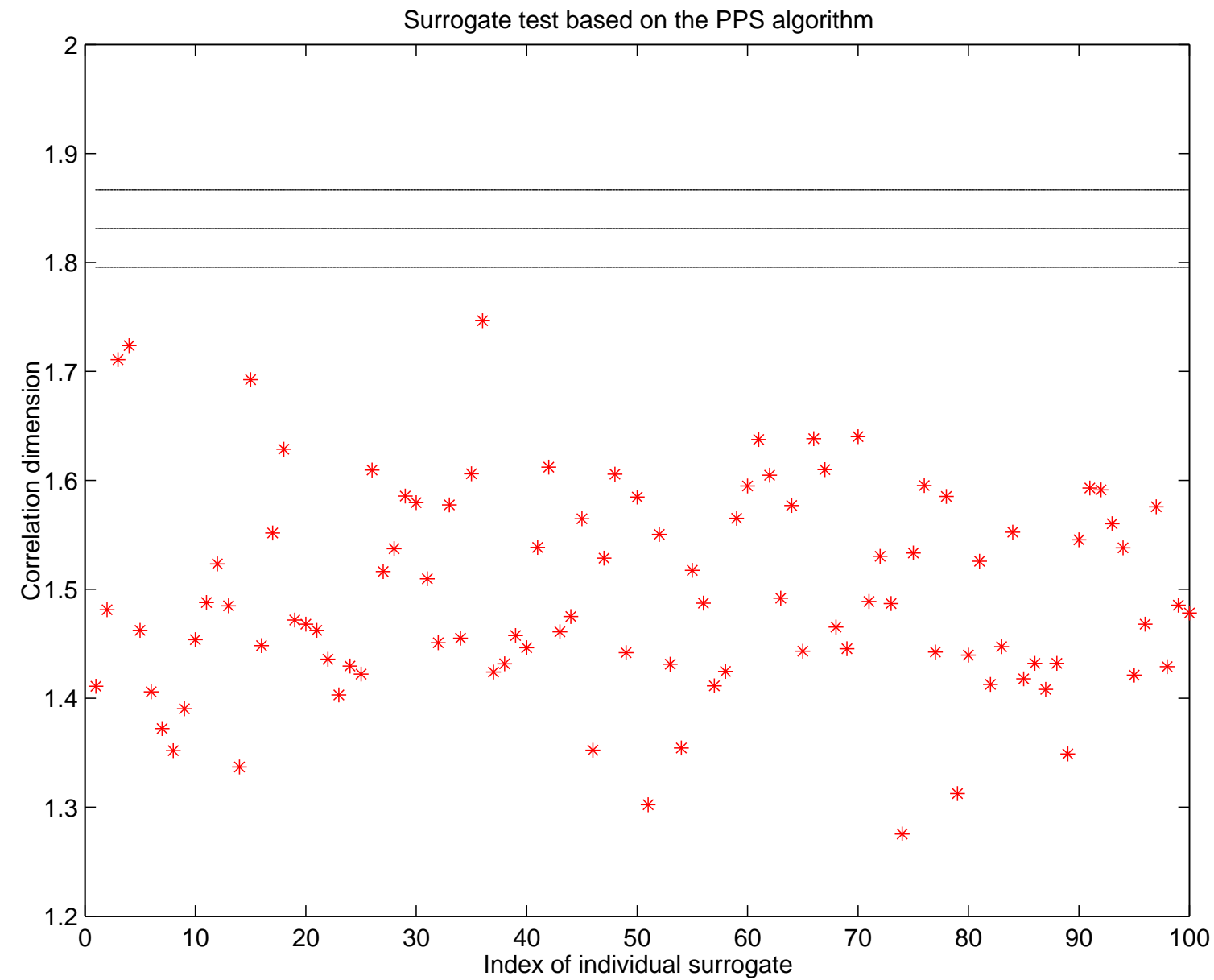

Figure 13: Surrogate test based on the PPS algorithm for the case of $a=0.1$ and $b=1.215$ with initial condition $(-0.5,6,1.1)$. The meaning of the lines and asterisks are the same as that in Fig. 4. 


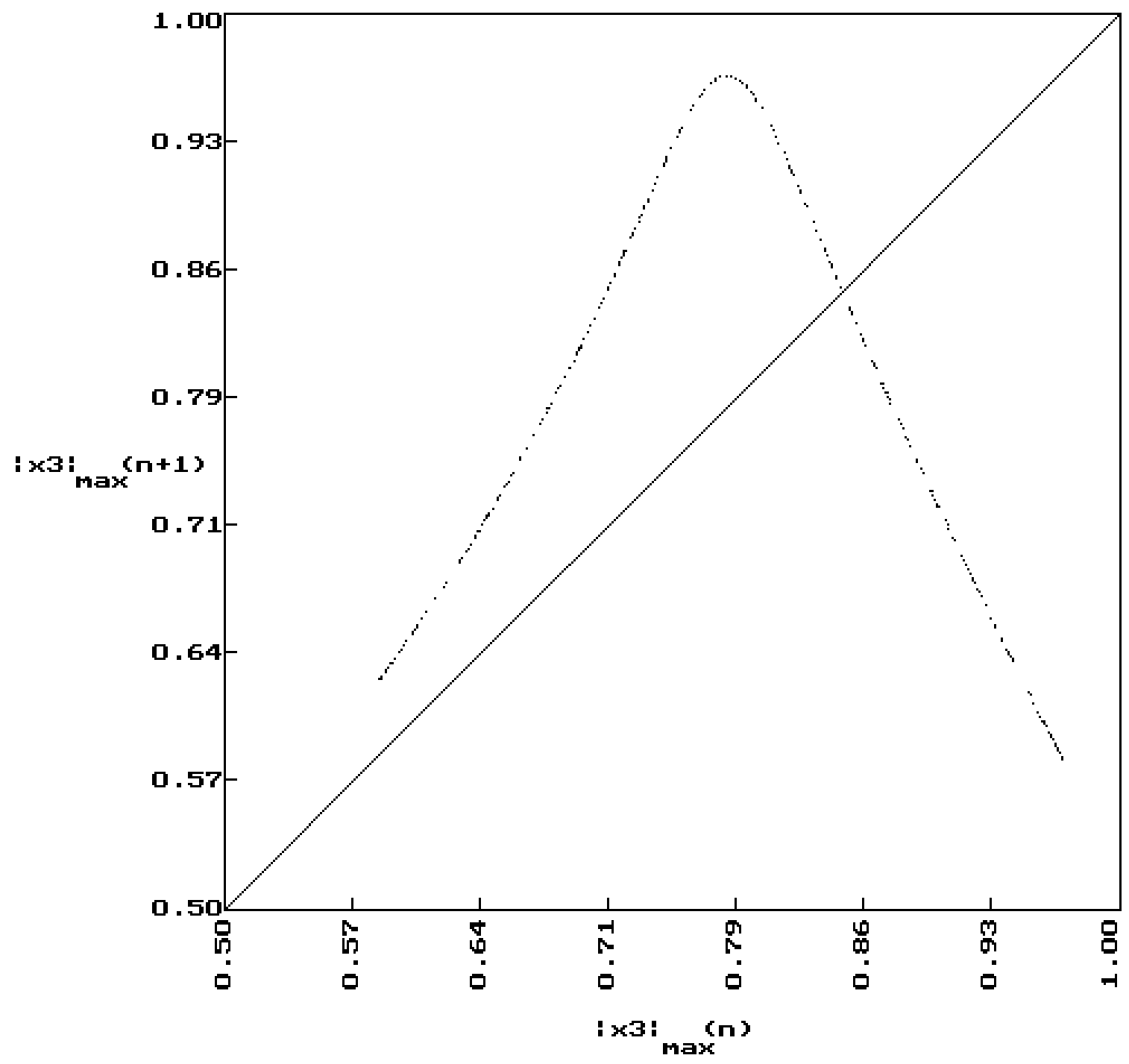

Figure 14: Next amplitude plot of the relative maximums for the case of $a=0.1$ and $b=1.215$ (taken from [Danca \& Chen 2004]). 
(a)

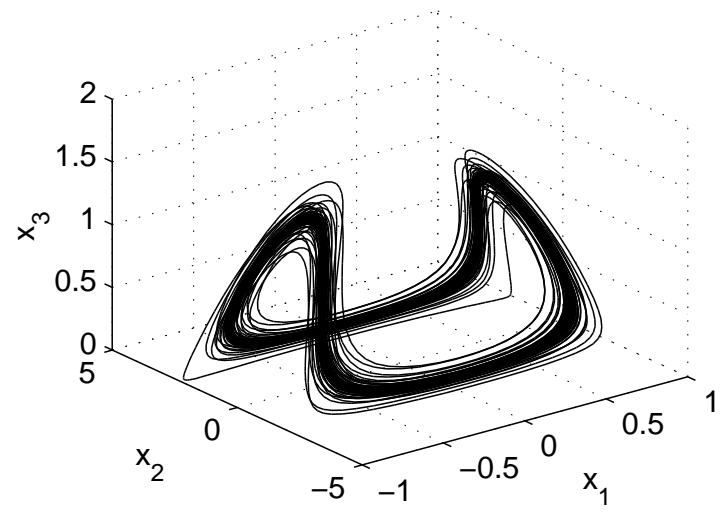

(c)

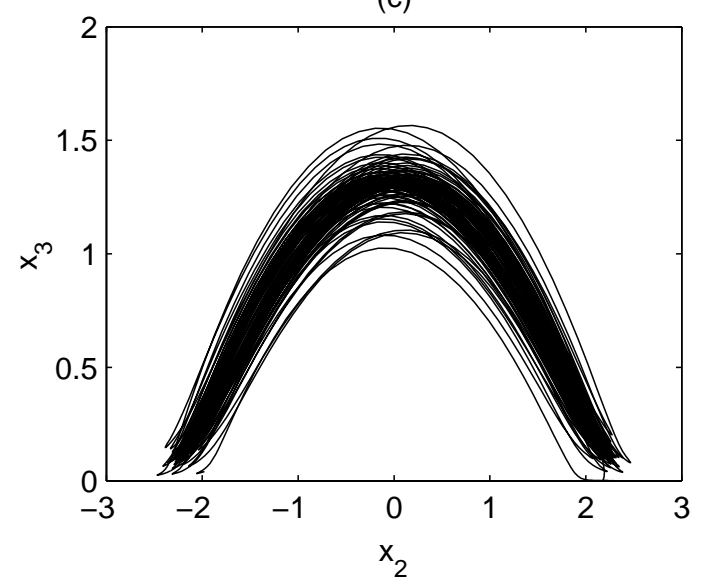

(b)

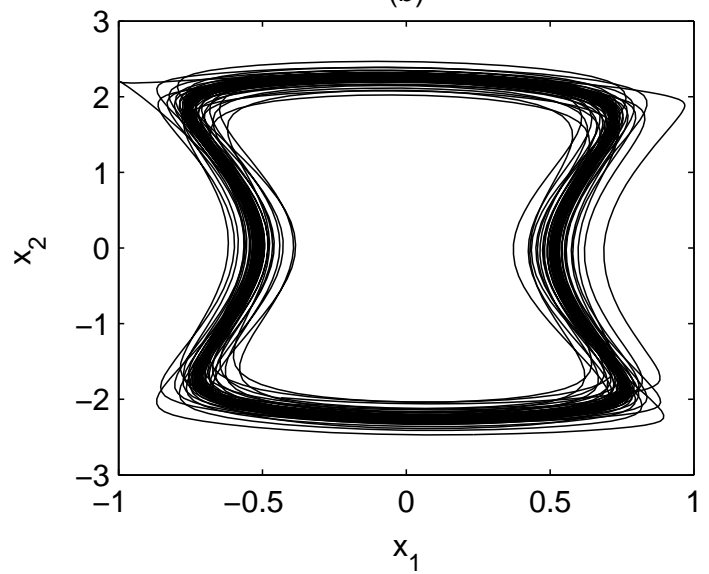

(d)

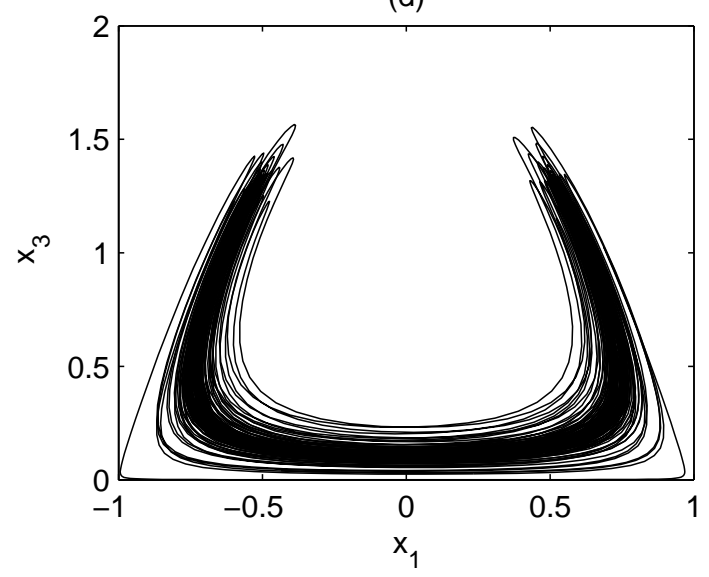

Figure 15: The chaotic attractor $A_{5}$ for the case of $a=0.05$ and $b=0.06$. (a) Three-dimensional view; (b)-(d) Two-dimensional views. 


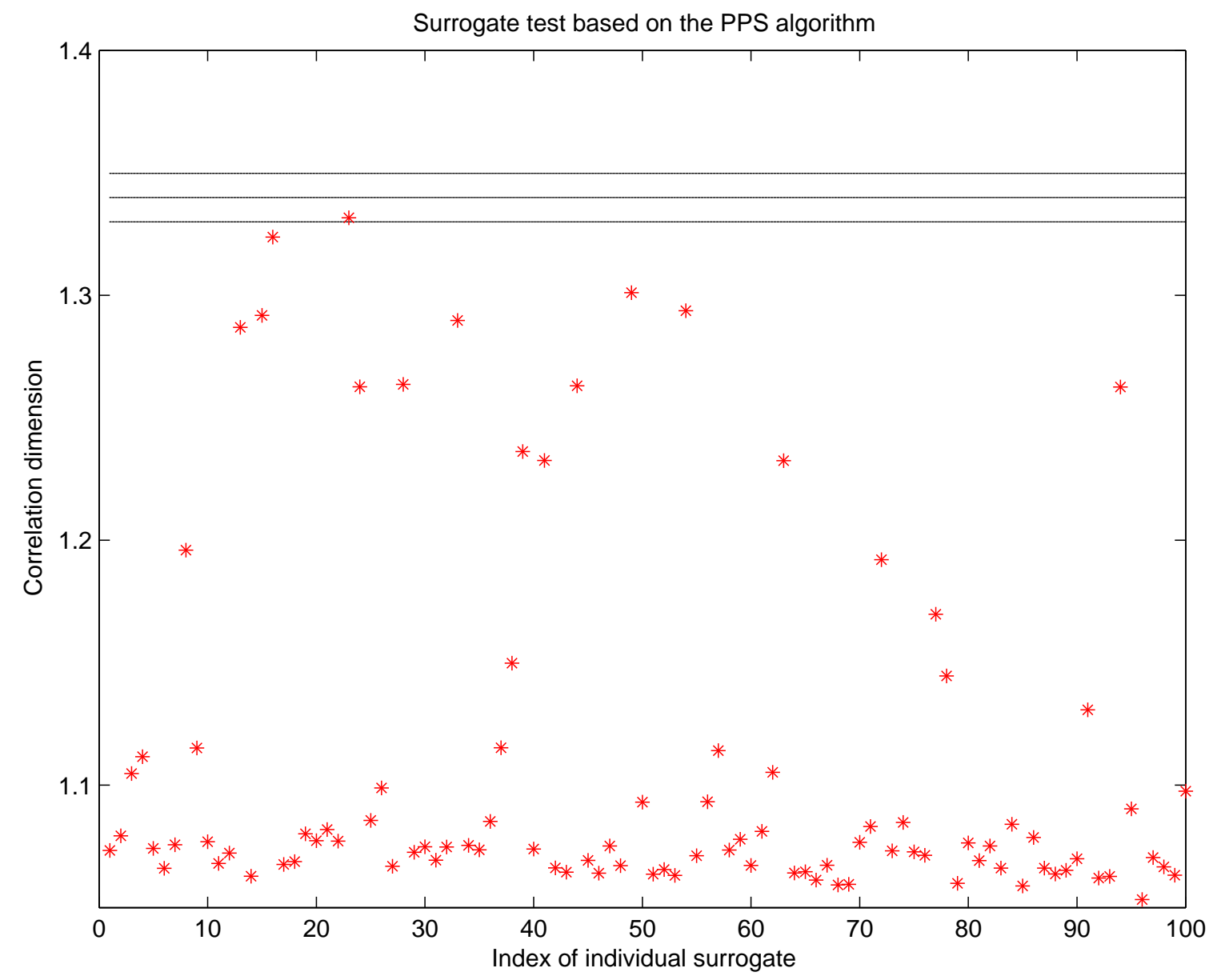

Figure 16: Surrogate test based on the PPS algorithm for the case of $a=0.05$ and $b=0.06$. The meaning of the lines and asterisks are the same as that in Fig. 4. 


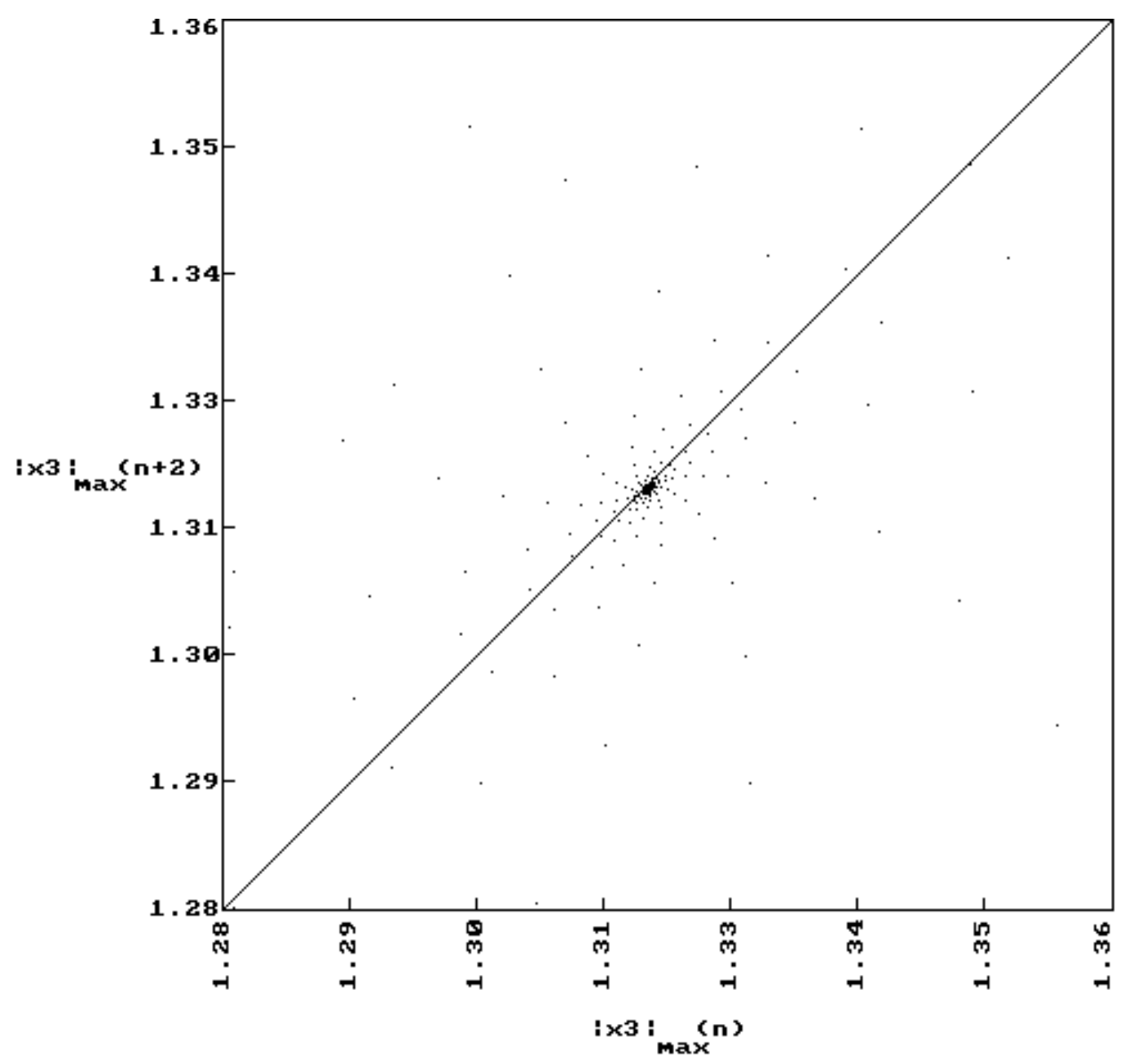

Figure 17: Next amplitude plot of the relative maximums for the case of $a=0.05$ and $b=0.06$. 
(a)

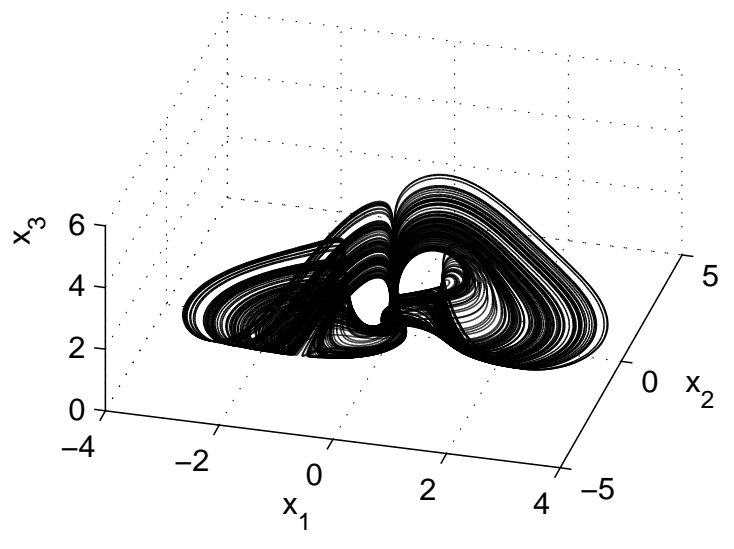

(c)

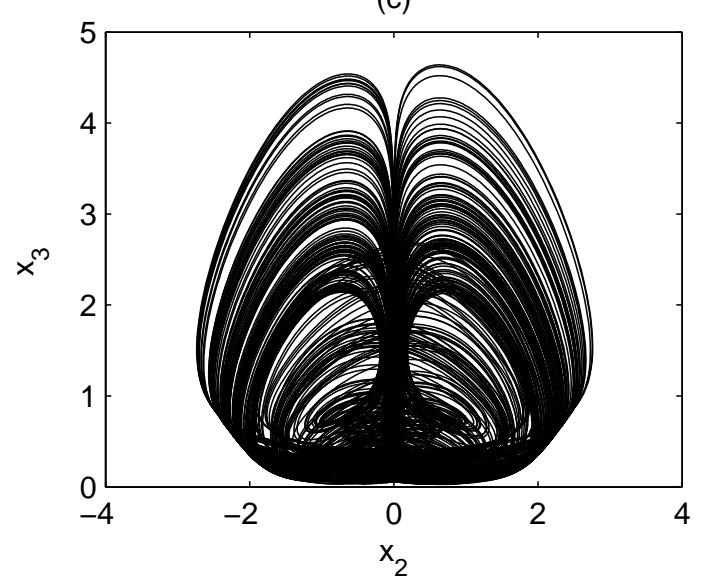

(b)

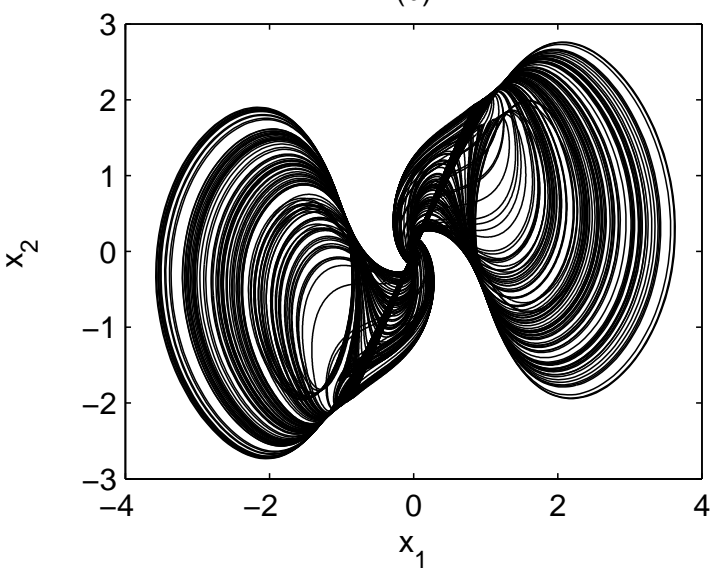

(d)

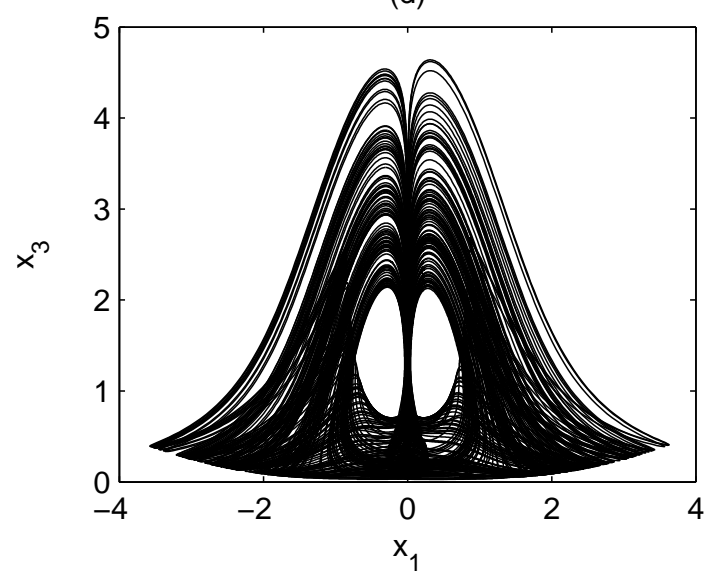

Figure 18: The chaotic attractor $A_{6}$ for the case of $a=-1$ and $b=-0.1$. (a) Three-dimensional view; (b)-(d) Two-dimensional views. 


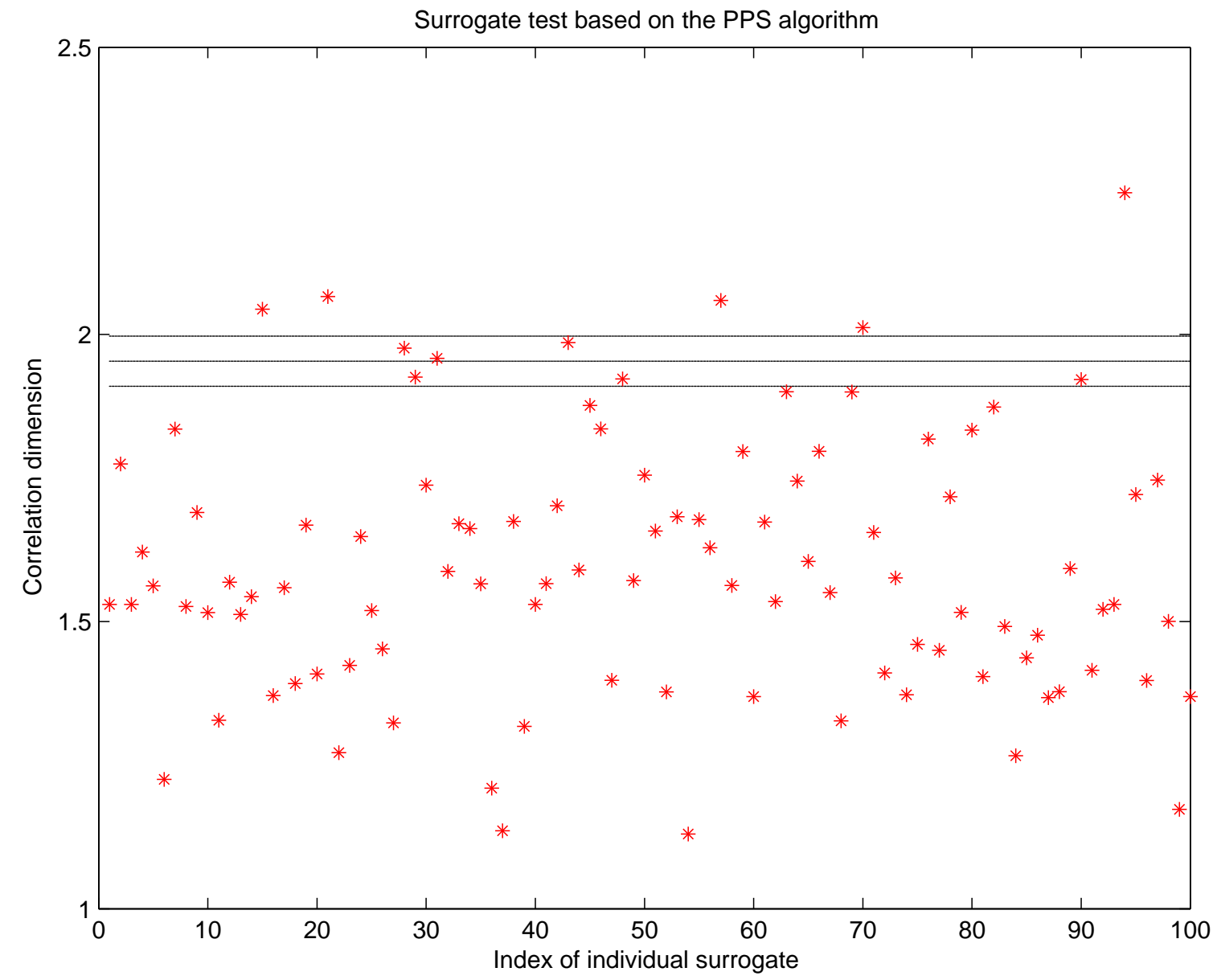

Figure 19: Surrogate test based on the PPS algorithm for the case of $a=-1$ and $b=-0.1$. The meaning of the lines and asterisks are the same as that in Fig. 4. 


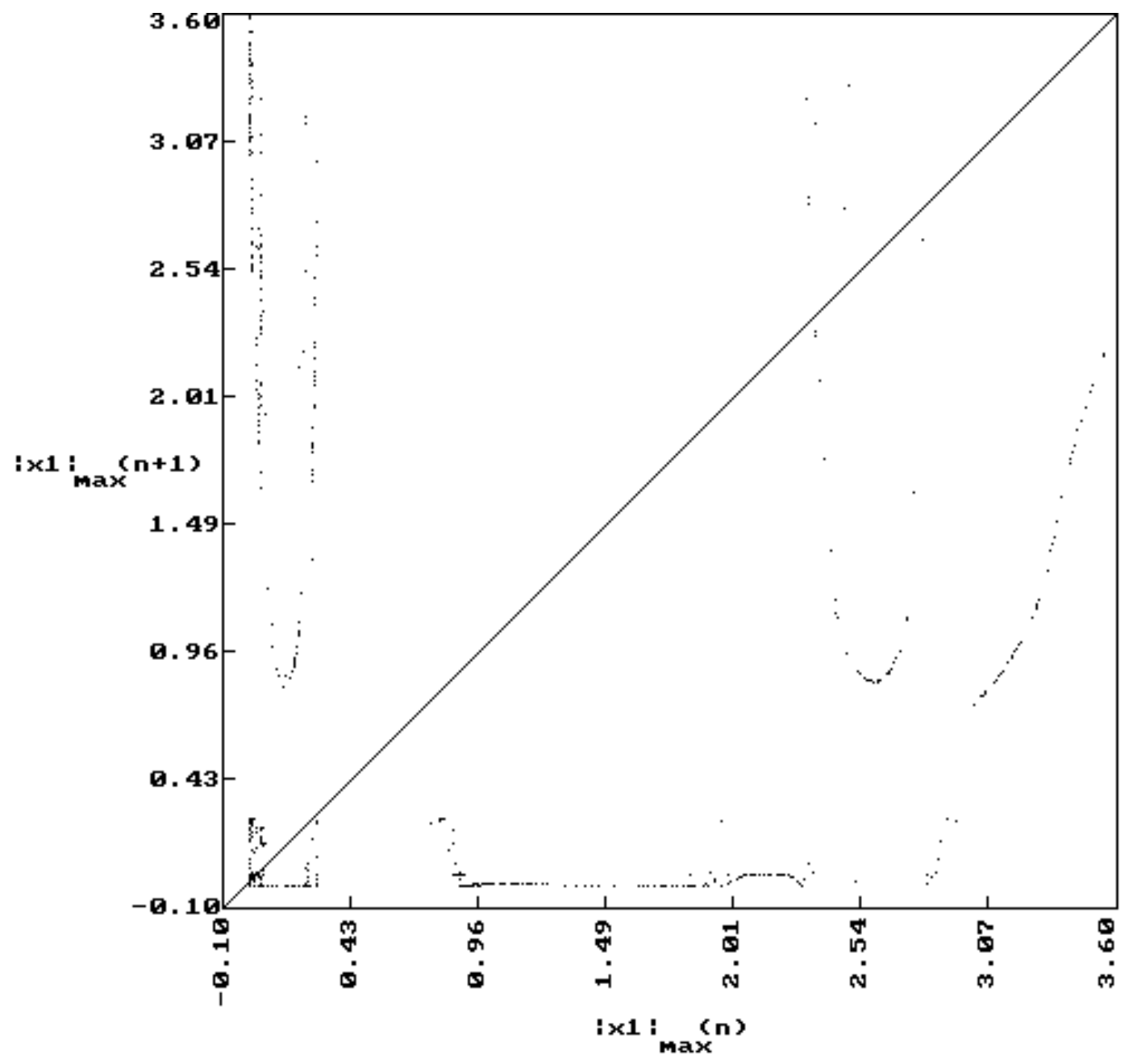

Figure 20: Next amplitude plot of the relative maximums for the case of $a=-1$ and $b=-0.1$. 


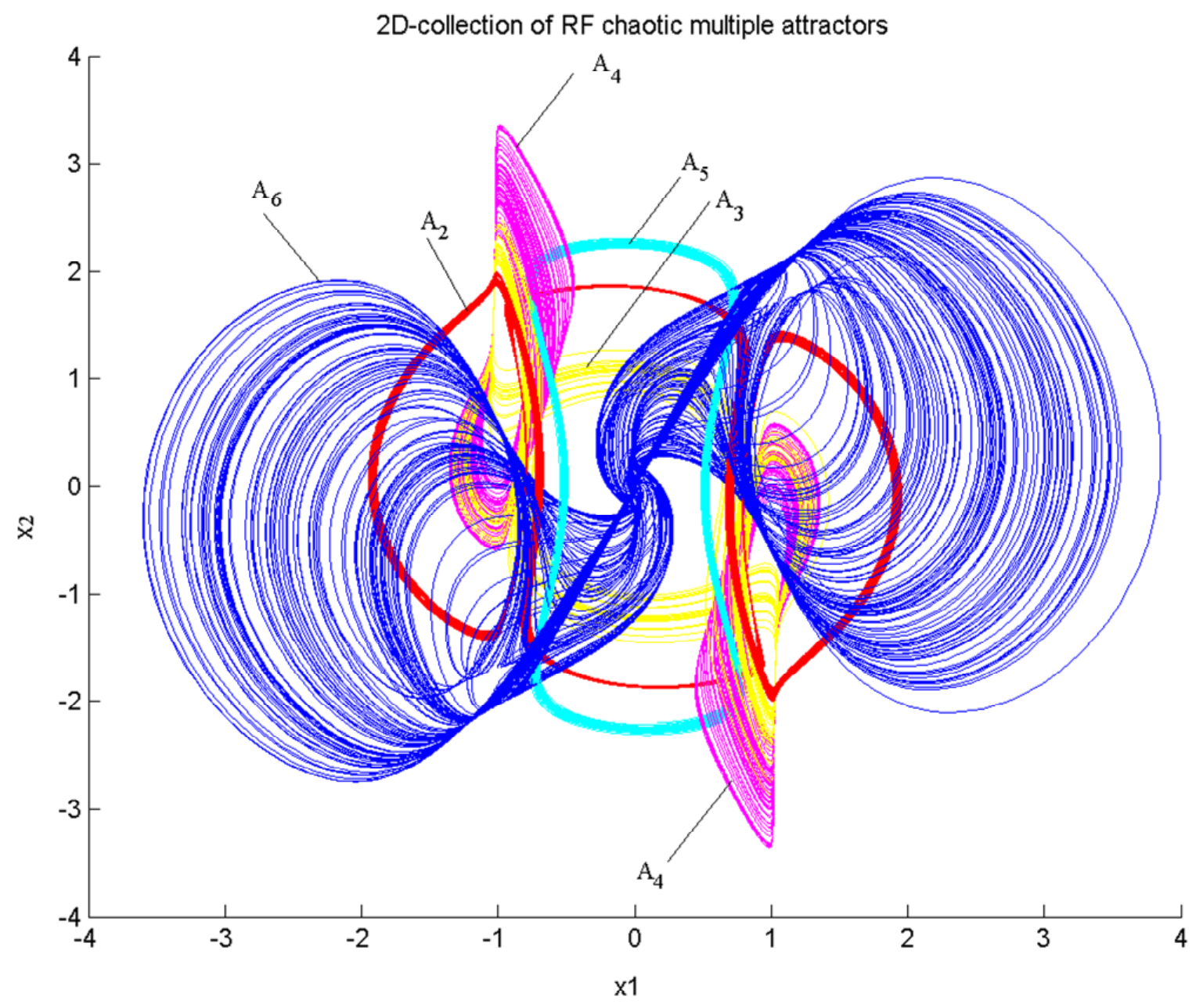

Figure 21: Two-dimensional collection of the multiple chaotic attractors $A_{1}-A_{6}$. 